NISTIR 6622

\title{
A Two-Phase Airframe Shielding Performance Study Using Ultra-wideband Measurement Systems
}

Chriss. A. Grosvenor Robert T. Johnk David R. Novotny Nino Canales Claude M. Weil Jason Veneman

\footnotetext{
QC 100 . 456 * 6622 2003 C. 2 



\title{
A Two-Phase Airframe Shielding Performance Study Using Ultra-wideband Measurement Systems
}

\author{
Chriss. A. Grosvenor \\ Robert T. Johnk \\ David R. Novotny \\ Nino Canales \\ Claude M. Weil \\ Jason Veneman
}

Radio-Frequency Technology Division Electronics and Electrical Engineering Laboratory National Institute of Standards and Technology

325 Broadway

Boulder, Colorado 80305

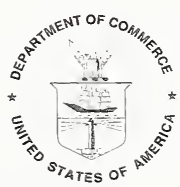

U.S. Department of Commerce Donald L. Evans, Secretary

Technology Administration Phillip J. Bond, Under Secretary of Commerce for Technology 



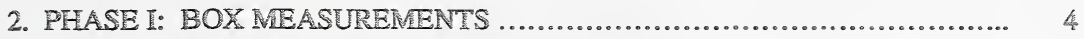

2.1 Measurement Systen for Collection of Bor. Data ......................... 5

2.2 Measurement Results ................................................. 6

2.2.1 Frequency Averaging ........................................ 6

2.2 .2 Paddle Averaging ............................................ ?

2.2.3 Angular Averaging ............................................ 7

2.3 Corrections ...................................................... 7

2.4 Conclusions for the Phase I Bor Effort ................................. 8

3. PHASE II: AIRCRAFT MEASUREMENTS ................................ 19

3.1 Measurement System for Aircraft Measurements ......................... 19

3.2 Extrapolation Measurements .......................................... 21

3.3 Passenger Deck (Center) Measurements .................................. 22

3.4. Passenger Deck (First Class) Measurements .............................. 23

3.5 Cockpit Measurements ............................................... 23

3.6 Cargo Bay Measurements ............................................... 23

3.7 Avionics Bay Measurements .......................................... 24

3.8. Comparison of Different Compartments ................................ 24

3.9 Summary of Direct Mumination Aircraft Measurements .................... 24.

3.10 The Reverberant Reference Method ..................................... 25

3.11 Uncertainty Analysis ............................................... 27

3.12 Conclusions for the Phase II Aircraft Effort ........................... 28

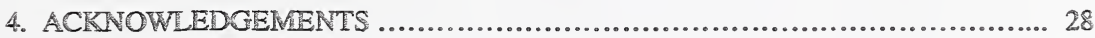

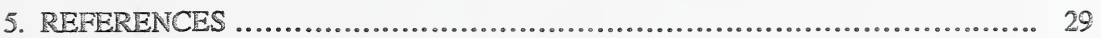





\title{
A Two-Phase Airfirame Shielding Performance Study Using Ultra-wideband Measurement Systems
}

\author{
Chriss A. Grosvenor \\ Robert T. Johnk \\ David R. Novotny \\ Nino Canales \\ Claude M. Weil \\ Jason Veneman \\ National Institute of Standards and Technology \\ Radio-Frequency Fields Group, 813.02 \\ 325 Broadway \\ Boulder, CO 80305
}

The National Institute of Standards and Technology participated in a comprehensive study of electromagnetic aircraft shielding. The effort consisted of two parts: (1) shielding measurements on a box model with a simple and precisely controlled geometry, and (2) a shielding evaluation of a Boeing 737-800 commercial airliner. The box measurements provided a baseline with which to study and to compare different shielding measurement techniques. A stepped-CW measurement system based on a vector network analyzer was used to perform direct-illumination measurements on the box, and to acquire a direct antenna-to-antenna coupling reference. The box measurements demonstrate that our measurement methods work well in cluttered environments and, with suitable correction factors, correlate well with reverberation chamber and anechoic chamber techniques. A direct-pulse, timedomain system based on a high-speed pulse generator, an ultra-wideband sampling oscilloscope, and NIST-developed linear-phase TEM horns were used to perform aircraft measurements. Two measurement procedures were invoked: (1) A directillumination method with the transmitter placed in various compartments, combined with a direct antenna-to antenna reference measurement, and (2) a new directillumination method with the transmitter placed in various compartments, combined with a reference measurement that exploits the reverberation characteristics of the hanger in which the aircraft is placed. The latter we refer to as the Reverberant Reference Method. The aircraft measurements demonstrate the ability of our measurement system to effectively perform in-situ aircraft shielding measurements in cluttered environments. This system should be of great interest to the military and commercial aircraft-testing communities.

Key Words: Aircraft shielding, antenna measurements, direct illumination, shielding effectiveness, time-domain measurements, UWB measurements 
The Time-Domain Free-Field Metrology (TDFFM) project team of the National Institute of Standards and Technology (NIST) participated in a joint effort to develop accurate, efficient, and low-cost aircraft shielding measurement techniques. The other participants in this effort were: (1) the Boeing Aircraft Company of Seattle, Washington, and (2) the Naval Surface Warfare Center (NSWC) of Dahlgren, Virginia. Each participant brought a unique set of skills and expertise to this effort. This measurement program is driven by an industry need for fast, accurate, and convenient tools to measure aircraft shielding performance in situ. This is a formidable metrological challenge since it requires a measurement system to perform in a variety of indoor and outdoor environments: aircraft production lines, hangars, flight lines, and other deployment scenarios. This particular effort consisted of two parts: (1) an initial validation phase consisting of shielding effectiveness (SE) measurements on a scale-model box, and (2) insitu measurements on a Boeing 737-800 jet in a hangar environment.

The scale-model is a precisely fabricated aluminum box with randomly placed circular apertures, designed to emulate the shielding and cavity characteristics of actual aircraft. It was empirically developed jointly by NIST and NSWC to study aircraft shielding performance using reverberation chamber and direct illumination techniques. A stepped-CW measurement system, based on a combination of a vector network analyzer (VNA) and a pair of dual-ridged horn antennas was used to perform the scale model measurements in the $2 \mathrm{GHz}$ to $4 \mathrm{GHz}$ frequency range. Shielding data were obtained using a two-step measurement process in which a directcoupling antenna reference is first acquired with boresighted antennas at a fixed separation, followed by a transmission measurement with the transmitting antenna placed inside of the box. A comparison of the data obtained from the two measurements yields the shielding. Since the box is electrically large in the frequency range from 2 to $4 \mathrm{GHz}$, large and rapid variations of \pm 20 $\mathrm{dB}$ occur in the shielding data as a function of frequency. These are strongly dependent on both antenna location and the operating frequency. More meaningful results can be obtained by smoothing the data using an averaging process. Several options are available: (1) paddle averaging using a moveable paddle that was incorporated into the box, (2) frequency-averaging over a specified bandwidth, (3) angular averaging over a number of receiving antenna locations, and (4) performing averaging using various combinations of 1,2 , and 3 . All of these techniques provide effective smoothing of shielding data, but combinations of them provide a multiplicative smoothing effect.

The box was first deployed and measured in the NIST (Boulder) anechoic chamber. The anechoic chamber has a low level of environmental scattering and simulates an ideal environment for direct illumination measurements. The box and the associated measurement system were then re-deployed and measured in an aircraft hangar, located at the Boeing Company manufacturing facility in Renton, Washington. The hangar environment is complex with a high level of environmental scattering that can potentially introduce additional propagation paths between transmitter and receiver, and unknown systematic errors into the shielding measurements. A comparison of the two sets of measurements shows that environmental effects are small. This demonstrates the robustness of the NIST measurement system. All in all, the box 
measurements demonstrated that direct illumination techniques are an accurate and efficient tool for measuring shielding performance.

The second phase of this effort consisted of an extensive series of measurements performed on a 737-800 jet, which was being prepared for delivery. The measurements were performed in the same aircraft hangar as the earlier box evaluations. The reason for measuring the aircraft inside a hangar is three-fold: (1) to provide protection from weather and large temperature variations, (2) to exploit the hangar shielding properties to suppress potential interference to airport safety and navigation systems that could be generated by our measurement systems, and (3) the aircraft does not have to be moved to a specialized test site. Another factor that reduced the potential for harmful interference was the use of a direct-pulse measurement system, consisting of a highspeed, ultra-wideband impulse generator, a sampling oscilloscope with a $12 \mathrm{GHz}$ bandwidth, and NIST-developed TEM (transverse electromagnetic) homs enabling transmission and reception in the $25-1000 \mathrm{MHz}$ frequency range. This system provides low radiated emissions with virtually no potential for interference. A synchronized, repetitive generator and waveform averaging suppresses ambient signals (e.g. television, FM radio, mobile radios, cellular telephones, etc.) making the system ideal for this application.

The measurement process was similar to that of the box. It consisted of a combination of a reference measurement using boresighted antennas external to the aircraft, followed by a shielding measurement with the transmitter inside the aircraft. Frequency-domain quantities were obtained by Fast-Fourier transforming (FFT) the oscilloscope waveforms. A time gate was applied to the reference waveform in order to isolate the direct antenna-to-antenna coupling and eliminate the effects of environmental scattering. A comparison of the two measurements provides the necessary components for computing airframe shielding. The transmitter was placed in four compartments: the passenger deck (center and first class locations), the cockpit, the cargo bay, and the avionics bay. Placing the transmitter inside the aircraft provided additional protection against interference by taking advantage of the airframe's shielding properties. The receiving antennas were placed at a number of aspect angles and distances outside of the aircraft. Simultaneous measurements were obtained at the receiving end in vertical and horizontal polarization using a combination of a 2-channel oscilloscope and two TEM horn antennas, doubling the efficiency of data acquisition-a vital enhancement in lieu of tight time constraints. An additional enhancement was achieved through the exclusive use of frequency averaging of shielding data, which, as the box validation measurements will show, eliminates the need for a paddle, and reduced the required measurement time by an order of magnitude.

The direct-pulse system performed well and yielded excellent results. The shielding data obtained showed a strong functional dependence with frequency, aspect angle, and distance. The cargo bay exhibited the highest shielding with values ranging from 23-30 dB. Minimum performance was obtained in the cockpit with values ranging from 9-20 dB. In order to address concerns about potential cavity coupling between the airframe and the hangar, a new way of performing reference measurements was developed and tested. In this method the earlier procedure for obtaining a reference was inverted by placing the transmitting and receiving antennas at a large separation in the hangar in such a way to maximize environmental effects and 
eliminate direct antenna-to-antenna coupling. This technique, referred to as the Reverberant Reference Method, uses the reverberant energy in the hangar as basis of comparison for computing shielding. The results obtained using this method were excellent and did not exhibit some of the anomalies that were observed at some receiving antenna locations. All in all, the results obtained demonstrate that the NIST-developed measurement systems are capable of performing aircraft shielding measurements in situ.

This report consists of two primary parts. The first part, sections $2.0-2.4$, describes the scalemodel box measurement effort, and provides a detailed summary of measurement methods and results obtained. Excellent box measurement results were obtained with shielding ranging from 25 to $35 \mathrm{~dB}$ in the 2 to $4 \mathrm{GHz}$ frequency range. A comparison is provided with reverberation chamber results obtained by Ladbury, Lehman and Koepke [1]. After correction factors are applied to account for differences in the two methods, good correlation is seen, with typical observed discrepancies in shielding on the order of $3 \mathrm{~dB}$.

The second half of this report, covered in sections 3.0-3.10, summarizes a series of shielding measurements performed on the 737-800 jet in a hangar. The reverberant reference technique is described in section 3.8, and the results look very promising. More evaluations of the reverberant reference method are currently being planned. The final section of the report provides a summary and conclusions.

\section{PHASE I: BOX MEASUREMENTS}

The first phase of this effort consisted of measurements on a scale model box, developed jointly by NIST and NSWC. We began the effort with a known artifact to validate our measurement concept, and to fine-tune the equipment and measurement procedure for optimal performance. The box was designed to simulate the shielding effectiveness of a commercial aircraft. The box, shown in figure 1, was designed with the following dimensions; $0.73 \mathrm{~m} \mathrm{x} 0.93$ $\mathrm{m} \times 1.03 \mathrm{~m}[2,3]$. The dimensions were selected to allow a sufficient modal density above 1 $\mathrm{GHz}$, which ensures reverberant behavior, and to simulate the behavior of a complex, isotropic, electrically large cavity. Aluminum was chosen for its fabrication ease, its light weight, and its high electrical conductivity. A paddle driven by a dc motor was installed within the cavity to provide a moveable boundary for mechanical mode-stirring. A magnet was attached to the paddle shaft and a compass installed on the outside of the box to monitor paddle movement. Five circular holes, each with a diameter of $1.6 \mathrm{~cm}$, were randomly drilled in each of the six sides of the box to provide internal-to-external coupling and to achieve shielding effectiveness values in the range of $20 \mathrm{~dB}$ to $30 \mathrm{~dB}$-typical for large commercial aircraft $[4,5]$. We measured the box in two different environments: (1) The NIST (Boulder) anechoic chamber, which is virtually reflection free, and (2) a large production hangar located at Boeing field in Renton, Washington, used to perform polishing and paint finishing of Boeing narrow-body jets ( $737 \& 757$ series) off the assembly line. The hangar is a large, all-metal building with an irregular shape and a reinforced concrete floor, and is an electrically large, complex cavity, with some reverberant behavior. With the anechoic chamber measurements providing the baseline, the hangar 
measurements allowed us to assess the impact of environmental scattering and hangar reverberation.

\subsection{Measurement System for Collection of Box Data}

The measurement system, used for the box measurements, is shown in figure 2. The components include a vector network analyzer (VNA) for the stepped-CW signal, two $20 \mathrm{~m}$ cables, and two double-ridged waveguide antennas. The VNA is configured to acquire complex transmission data ( $S_{12}$, magnitude and phase). Prior to connecting the cables to the antennas, a two-port electronic calibration kit, using a full 12-term error model, was used to minimize system uncertainties due to cable phase characteristics, cable losses, and connector mismatch errors. This step provides a precisely calibrated system at the antenna terminals, with high stability, accuracy, and large dynamic range.

The extraction of box shielding characteristics requires the two-step measurement process, shown in figure 2. In the first step a reference measurement is performed by boresighting the transmitting and receiving antennas at a chosen, fixed distance that is the same for each step, and a transmission measurement is taken. This measurement gives us a measure of the energy incident on the box and it enables us to calibrate out the frequency-dependent effects of the antennas. The signal processing sequence is shown in figure 3. An Inverse Fast-Fourier transform (IFFT) is applied to the transmission data to obtain a time-domain waveform. In order to suppress ground bounce effects, room reflections, and to isolate the direct antenna-to-antenna coupling, a time gate is applied to the waveform. A Fast-Fourier Transform (FFT) is applied to the gated waveform to obtain a free-space antenna-to antenna reference. In the second measurement, the transmitting antenna is placed inside the box, and another transmission measurement is made, quantifying box penetration. The shielding effectiveness is defined as the ratio of the transmitted power of the antenna within the box to the transmitted power of the reference,

$$
S E=\frac{\mid\left. S_{12}(\text { box penetration })\right|^{2}}{\mid\left. S_{12}(\text { gated reference })\right|^{2}}
$$

where $\mid S_{12}$ (box penetration) $\left.\right|^{2}$ is the transmission amplitude spectrum with the transmitting horn located inside the box, and $\mid S_{12}$ (gated reference) $\left.\right|^{2}$ denotes the time-gated reference amplitude spectrum. This definition of shielding is the same as the one used for panel measurements of shielding materials [6]. A series of reference and penetration measurements were taken in the NIST anechoic chamber at distances of $1 \mathrm{~m}, 2 \mathrm{~m}, 3 \mathrm{~m}$, and $4 \mathrm{~m}$ at angular increments of 20 degrees. A measurement setup with the box in the anechoic chamber is shown in figure 4 . These were taken before the measurements of the box in the hangar facility, and established a baseline data set in a near-perfect (anechoic) environment. A second series of measurements was then performed in the hangar. The test setup used is illustrated in figure 5. Due to time constraints, the distance from the receiving antenna aperture to the box center was fixed at $3 \mathrm{~m}$. This constraint also limited our taking of data with a vertically polarized receiving antenna. An 
azimuthal antenna scan was performed at discrete intervals of 30 degrees by manually positioning the receiving antenna over reference marks drawn on the hangar floor and boresighting it at the box center. The receiving antenna and the box center were oriented at a fixed height of $2 \mathrm{~m}$. Mechanical stirring was performed at each receiving antenna position by rotating the box paddle in discrete 60 degree steps. A transmission measurement was performed for each paddle position, resulting in a total of six transmission measurements for each azimuthal angle. A total of 72 measurements were taken in a scan. A photo of the box setup in the hangar is shown in figure 6, where the box is mounted on a low-density extruded polystyrene tower with $\varepsilon_{\mathrm{r}}=1.04$ to minimize box-mount tower interactions. Figures 7 and 8 show $200 \mathrm{MHz}$ segments of shielding data obtained at two different paddle positions. The large variations, typically $20 \mathrm{~dB}$, are due to the reverberant behavior of the box cavity. The rapid variations, resembling noise, in the transmission amplitude spectra indicate a large number of cavity modes; it is also interesting to note the graphs are quite similar at both paddle positions. A more detailed analysis using cross-correlation functions indicated that the transmission data at the two positions were virtually uncorrelated-indicative of effective mechanical stirring.

\subsection{Measurement Results}

Large variations in shielding data make it difficult to assign a value for shielding effectiveness, but we can look at various data processing techniques to smooth the data and obtain more meaningful results. The approach used here consists of performing a scalar power averaging of the shielding data, a classic reverberation technique. There are several ways to do this: (1) averaging with respect to frequency, (2) averaging with respect to paddle position, and (3) averaging over the angular positions of the receiving antenna. Dramatic enhancements can be obtained by combining two or more averaging techniques. The following sections discuss how we incorporated each technique and how ultimately we settled on a single technique to provide an average value for the shielding effectiveness of the box.

\subsubsection{Frequency Averaging}

Frequency averaging is one technique used for reverberation chamber measurements. In this technique, a sliding window of a specified bandwidth is used to perform an average and the window moved along to the end of the record;

$$
\left\langle S E\left(f_{n}\right)\right\rangle=\frac{1}{2 N+1} \sum_{i=n-N}^{n+N}\left|S E\left(f_{i}\right)\right|^{2},
$$

where the index $n$ is the center frequency of the averaging window, $N$ is the number of discrete FFT frequencies above and below the center frequency of the window, and $\left|\operatorname{SE}\left(f_{i}\right)\right|^{2}$ corresponds to scalar power. In this technique, the modal structure in the box changes with frequency, and thereby randomizes the field structure. Larger frequency bandwidths provide larger shifts in modal structure, producing a higher degree of smoothing. This method is an electronic mode stirring technique that does not require a paddle-making it time-efficient and well suited for high-efficiency aircraft measurements. The impact of increasing the averaging bandwidth is shown in figures 7, 9, 10 and 11 using $0 \mathrm{MHz}$ (no frequency averaging), $5 \mathrm{MHz}, 20 \mathrm{MHz}$, and 50 
$\mathrm{MHz}$ bandwidths, respectively. As shown in the figures, the wider the averaging bandwidth, the greater the smoothing - a result of increased randomization of the box modes. A comparison made between measurements taken in the NIST anechoic chamber and the measurements made in the hangar are shown in figure 12 with a $50 \mathrm{MHz}$ averaging bandwidth. Good correlation is seen with typical discrepancies no greater than $1-2 \mathrm{~dB}$, well within our measurement uncertainty to be discussed in the next phase.

\subsubsection{Paddle Averaging}

The next type of averaging is paddle averaging where we perform a scalar power averaging over all paddle positions at a fixed frequency. The following equation is used to average paddle positions:

$$
\langle S E(p)\rangle=\frac{1}{M} \sum_{i=1}^{M}\left|S E\left(p_{i}\right)\right|^{2},
$$

where the index $i$ denotes the paddle angle, and $M$ is the total number of paddle positions. The mechanical movement of the paddle changes the box boundary, shifts the modal structure, and provides a mechanism for randomizing the electromagnetic fields inside the box. This provides an effective basis for scalar power averaging and smoothing the shielding data. The equivalence [7] between paddle averaging and frequency averaging is shown in figure 13. The two traces show the similarity between averaging over a $50 \mathrm{MHz}$ bandwidth, with the paddle fixed, and the same measurement averaged over 6 paddle positions and no frequency averaging. No significant discrepancies are noted between the two methods, and only small differences in the frequencydependent fine structure are noted.

\subsubsection{Angular Averaging}

Angular averaging is performed using a scalar power average over all angular positions with the paddle position and frequency fixed.

$$
\langle S E(a)\rangle=\frac{1}{L} \sum_{l=1}^{L}\left|S E\left(a_{l}\right)\right|^{2},
$$

where $l$ denotes angular position and $L$ is the total number of angles measured. Figure 14 shows excellent agreement between paddle and angular averaging. Figure 15 shows the combination of all three types of averaging compared to the individual averaging techniques. One can see the convergence to an average shielding value as the three techniques are combined. However, since the three techniques have been shown to be equivalent, we can improve measurement efficiency by using frequency averaging alone.

\subsection{Corrections}

There are differences between reverberation-chamber measurements and direct- 
illumination measurements. These differences are a current research topic at NIST, but a brief discussion of the main components used to account for the differences is given here. In the direct-illumination technique, only one polarization at a time is measured, whereas; in the reverberation measurements both polarizations are measured simultaneously. In both measurements, we assume that the energy emanating from the box is composed equally of vertical and horizontal polarization. Since the direct-illumination method measures only one polarization, we account for this polarization mismatch $[8,9]$ which is half the energy or $3 \mathrm{~dB}$ in power. The other correction factor is for antenna gain. In reverberation-chamber measurements the measured power is independent of the antenna gain. However, outside of that environment the measured power is very much dependent on the antenna gain, by definition. Therefore, we must account for the antenna gain when comparing direct illumination measurements with reverberation-type measurements. A plot of the measured gain for a double-ridged antenna is shown in figure 16. Across the 2 to $4 \mathrm{GHz}$ band the antenna shows gain of around $9 \mathrm{~dB}$. The final corrected data for the box as compared to the anechoic chamber measurements is shown in figure 17. The anechoic data compares within 2 to $3 \mathrm{~dB}$ with measurements done in the NIST reverberation chamber.

\subsection{Conclusions for the $\mathbb{P}$ hase I Box $\mathbb{E}$ ffort}

In summary, the corrected shielding effectiveness of the box varies between $25 \mathrm{~dB}$ and 35 $\mathrm{dB}$ from $2 \mathrm{GHz}$ to $4 \mathrm{GHz}$, as shown in Figure 17 with uncertainties of $\pm 2 \mathrm{~dB}$. We looked at various averaging techniques and their combinations, to obtain an average shielding value for the box. Frequency-averaging was chosen because it provided the most smoothing with the greatest measurement efficiency. The original measurements were corrected to exclude the gain of the antenna and polarization mismatch. 


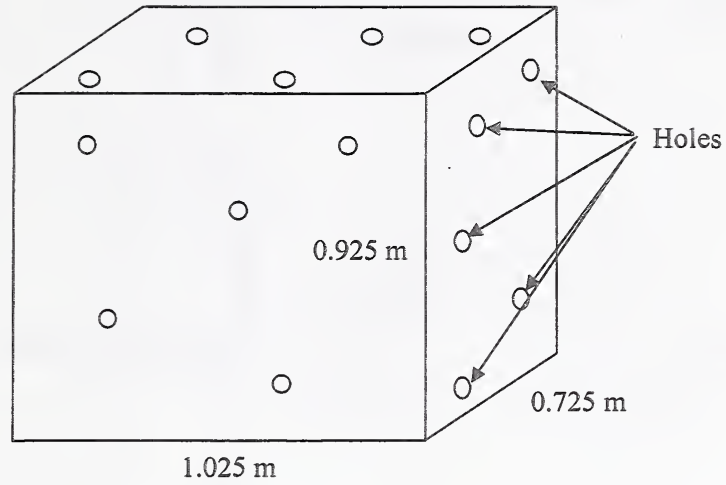

Figure 1. Dimensions of scale-model box artifact. 


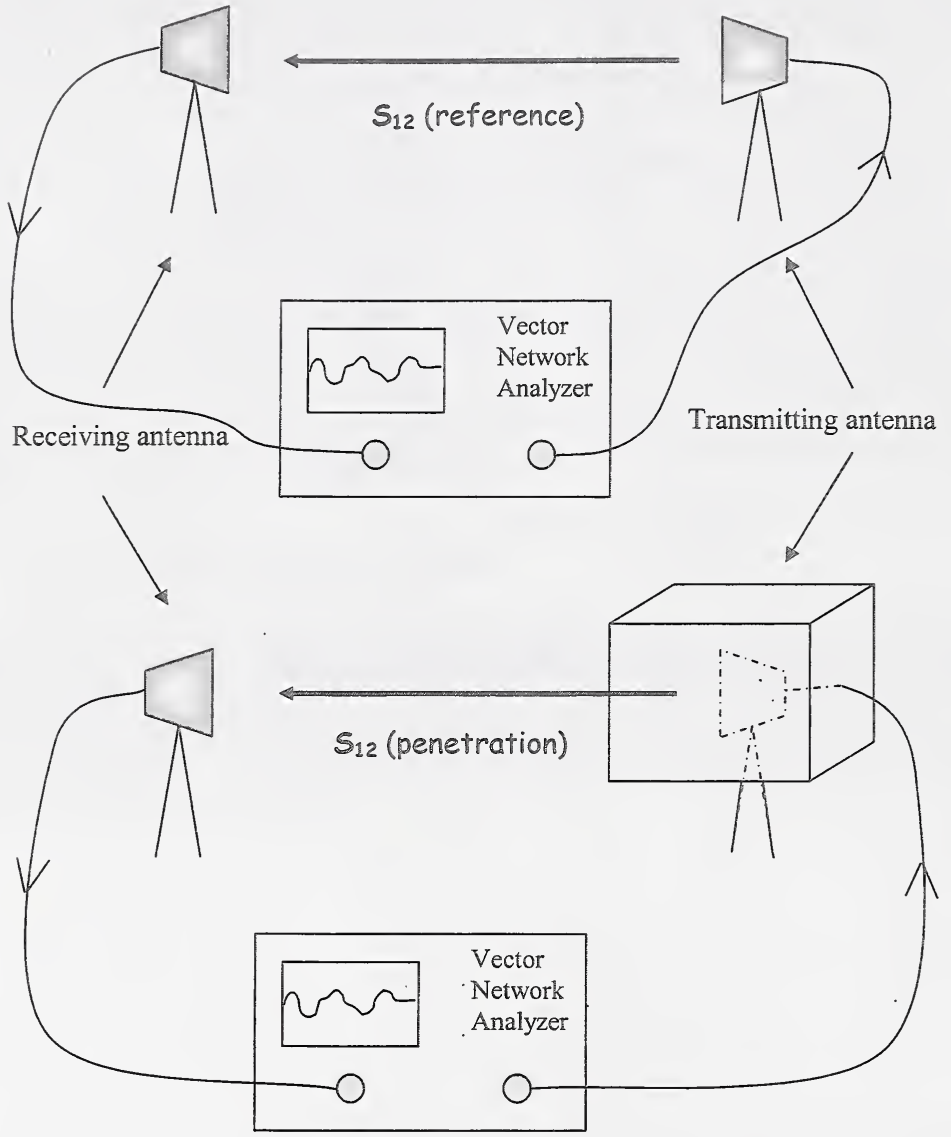

Figure 2. The upper drawing is a reference measurement showing system components, and the lower drawing a shielding measurement of the box. 


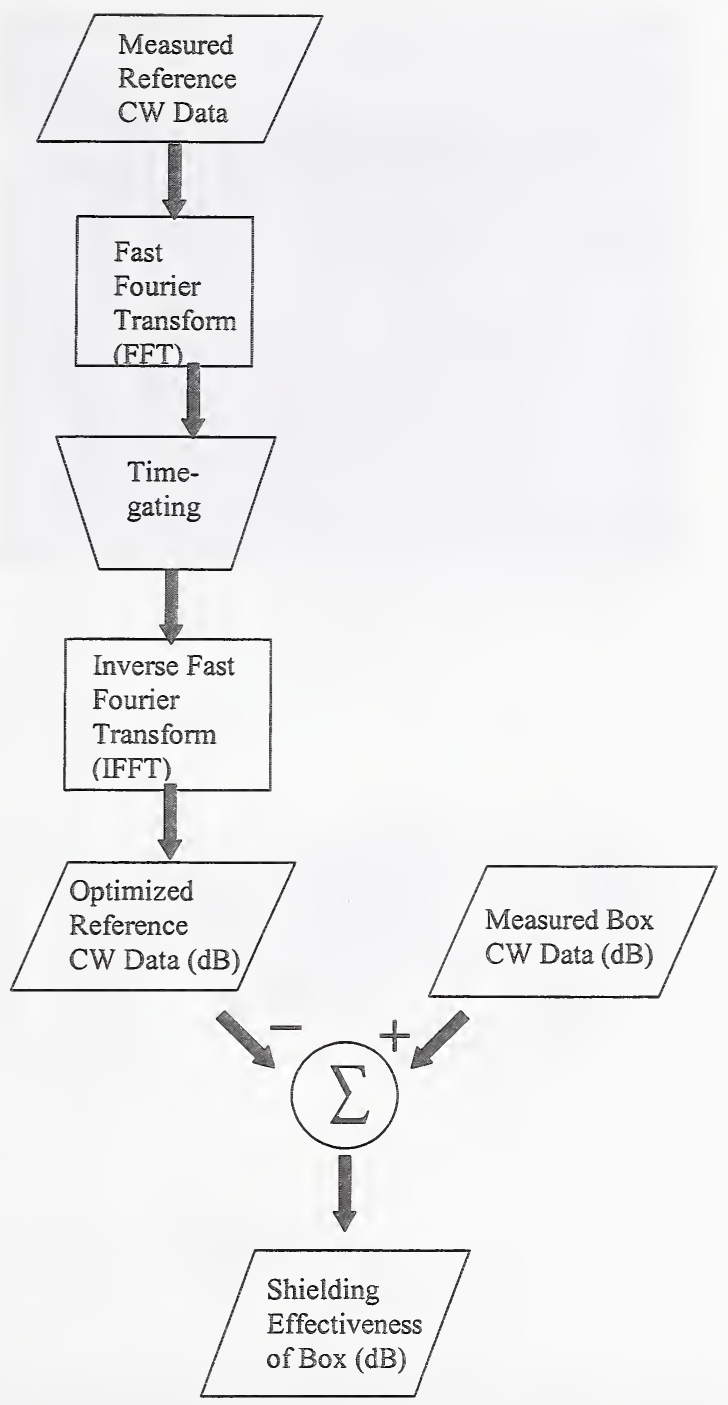

Figure 3. Signal-processing sequence to obtain the reference and the shielding values. 


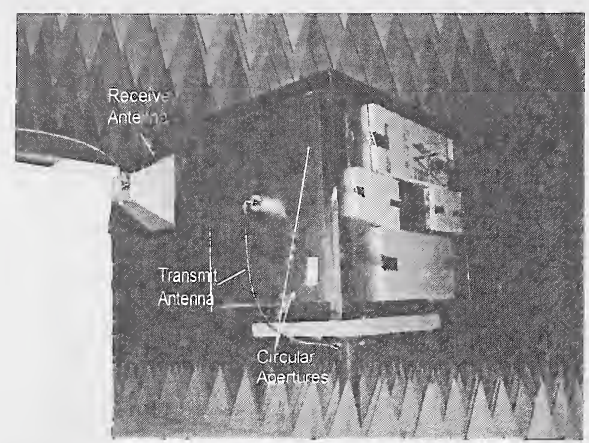

Figure 4. Box reference measurements in anechoic chamber at a separation of $1 \mathrm{~m}$.

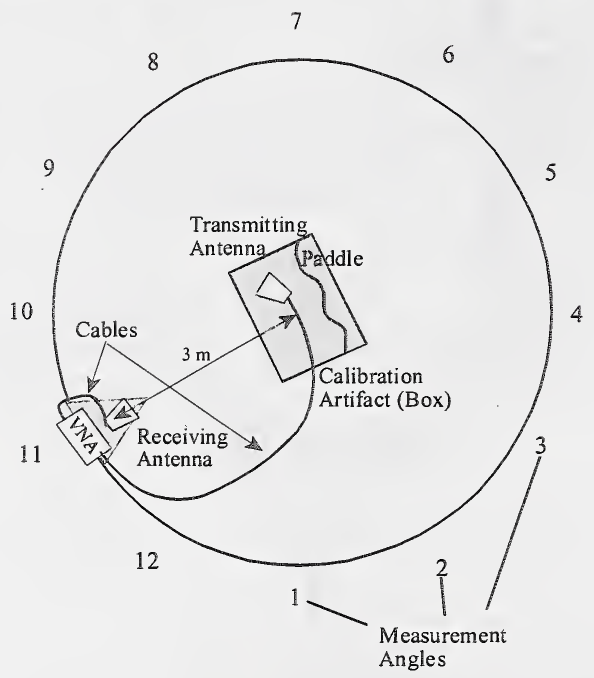

Figure 5. Box measurement setup at the Renton facility. A reference circle, $3 \mathrm{~m}$ in diameter, was drawn on the hangar floor with reference marks provided at 30-degree intervals for receiving antenna positioning. 


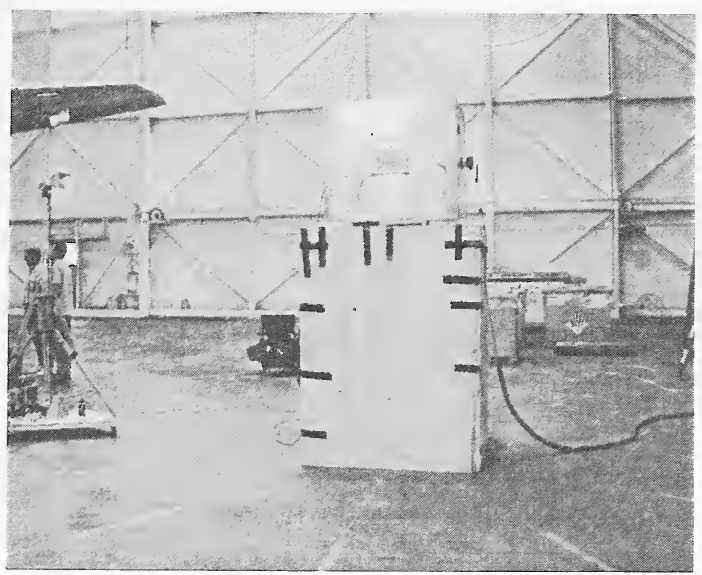

Figure 6. Box measurement at the Renton facility.

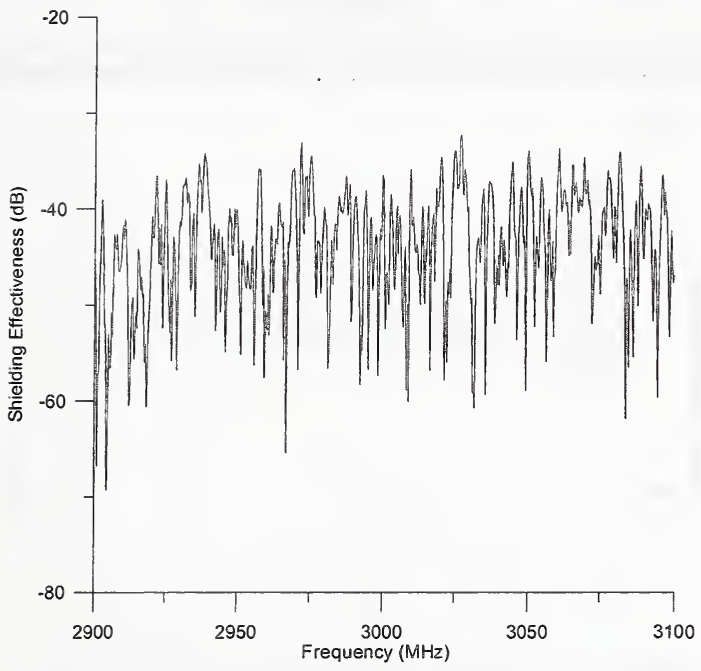

Figure 7. Shielding effectiveness (uncorrected) vs. frequency, for angle 1, paddle position 0. Note the large variations in shielding values when no averaging is used. 


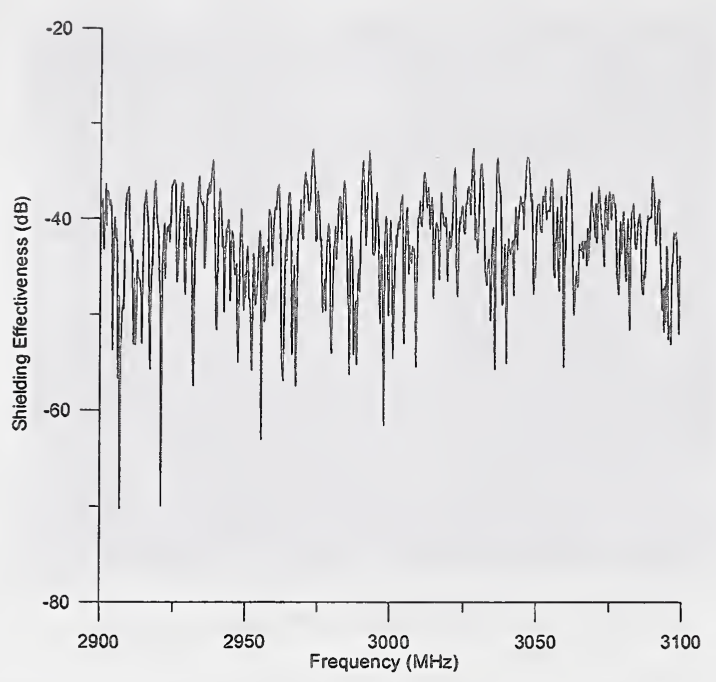

Figure 8. Shielding effectiveness (uncorrected) vs. frequency for angle 1, paddle position 1.

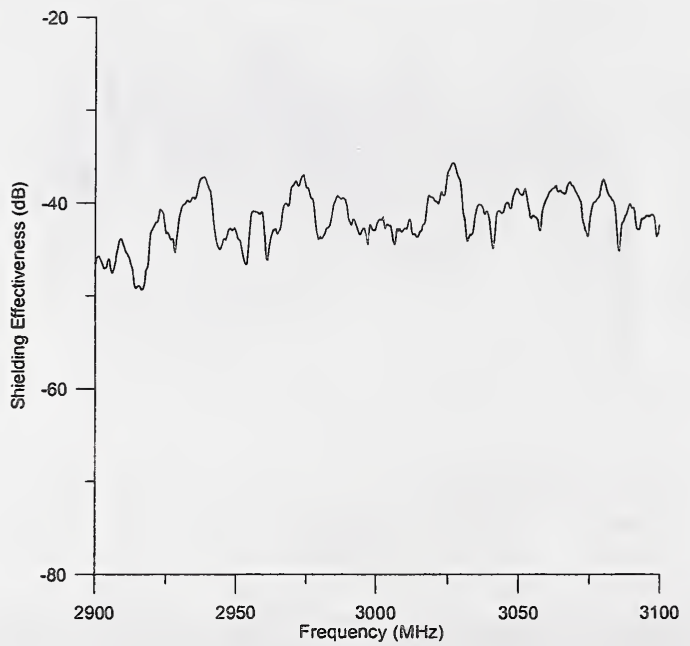

Figure 9. Frequency averaged shielding effectiveness (uncorrected) obtained with a $5 \mathrm{MHz}$ bandwidth at angle 1, paddle position 0 . 


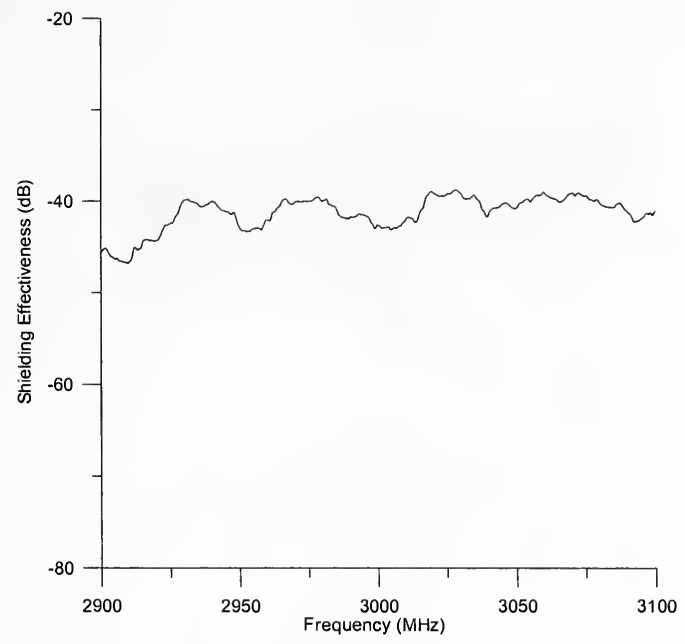

Figure 10. Frequency averaged shielding effectiveness (uncorrected) obtained with a $20 \mathrm{MHz}$ bandwidth at angle 1 , paddle position 0 .

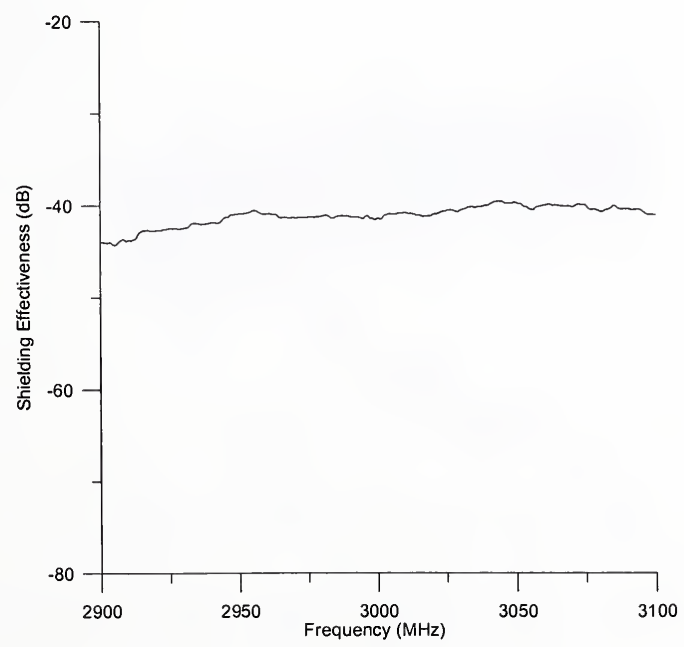

Figure 11. Shielding effectiveness (uncorrected) vs. frequency for angle 1, paddle position 0 , and $50 \mathrm{MHz}$ frequency averaging. 


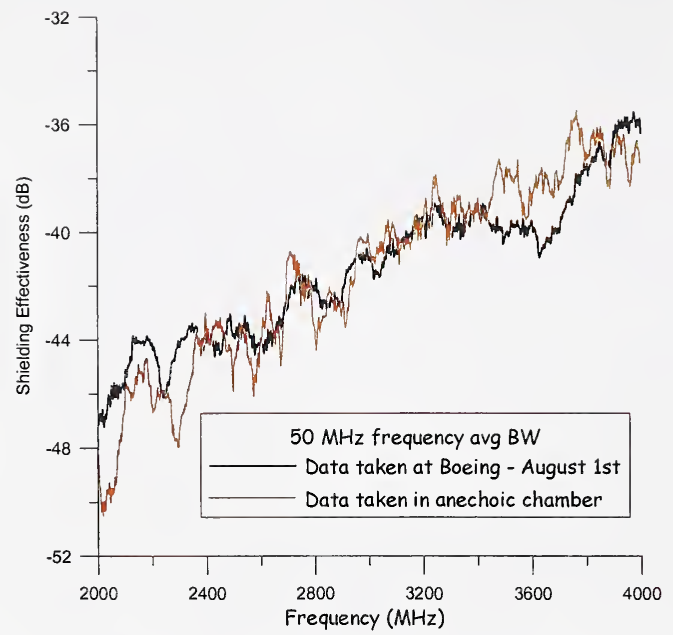

Figure 12. Comparison between data (uncorrected) taken on the box both in the anechoic chamber at NIST and the aircraft hangar.

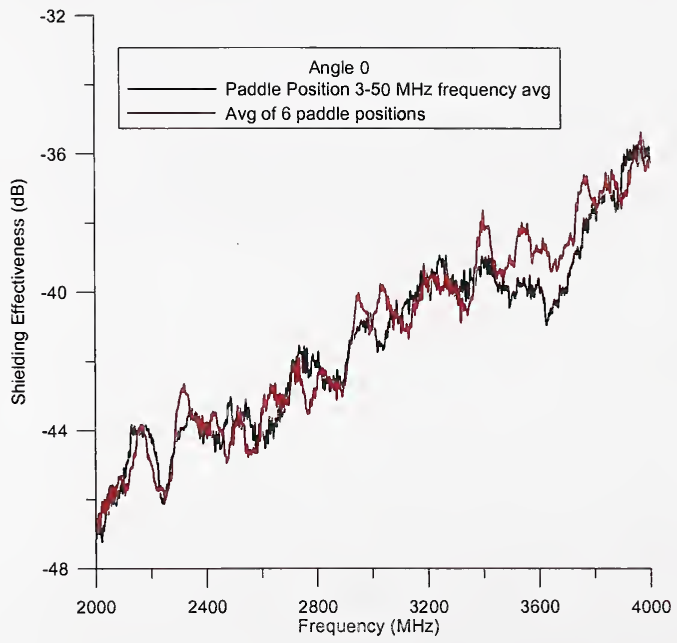

Figure 13. Comparison of shielding effectiveness (uncorrected) for frequency averaging (black line) and paddle averaging (red line). 


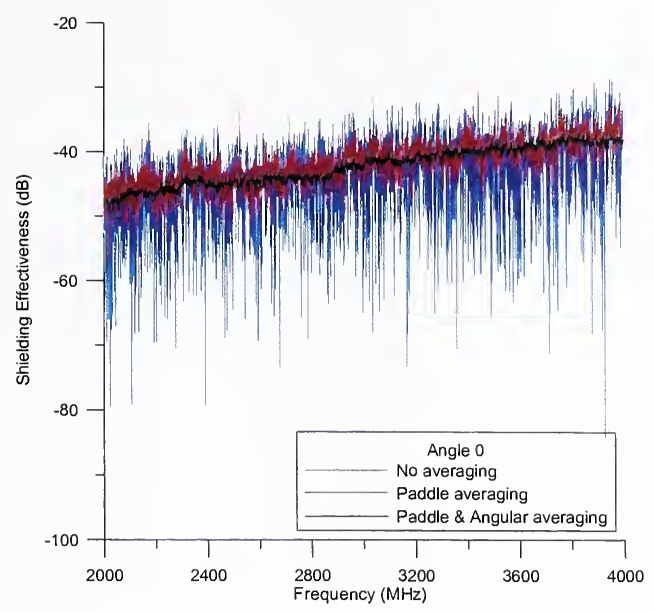

Figure 14. Comparison of shielding effectiveness (uncorrected) with paddle and angular averaging.

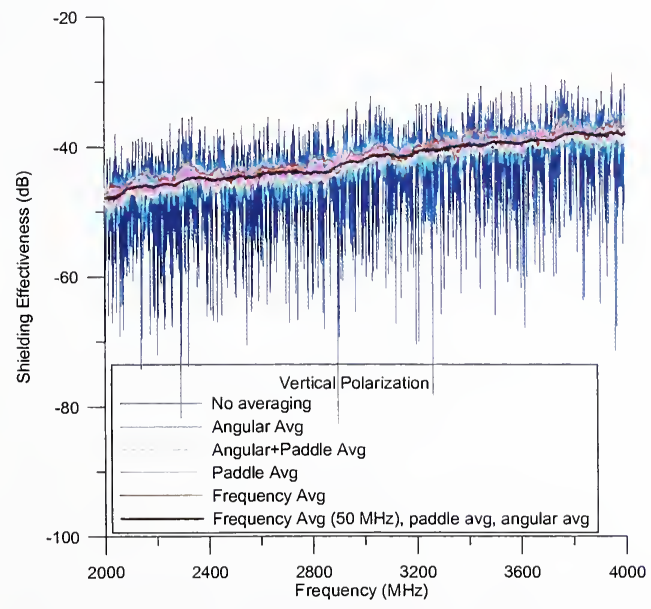

Figure 15. Comparison of various averaging techniques for uncorrected data. 


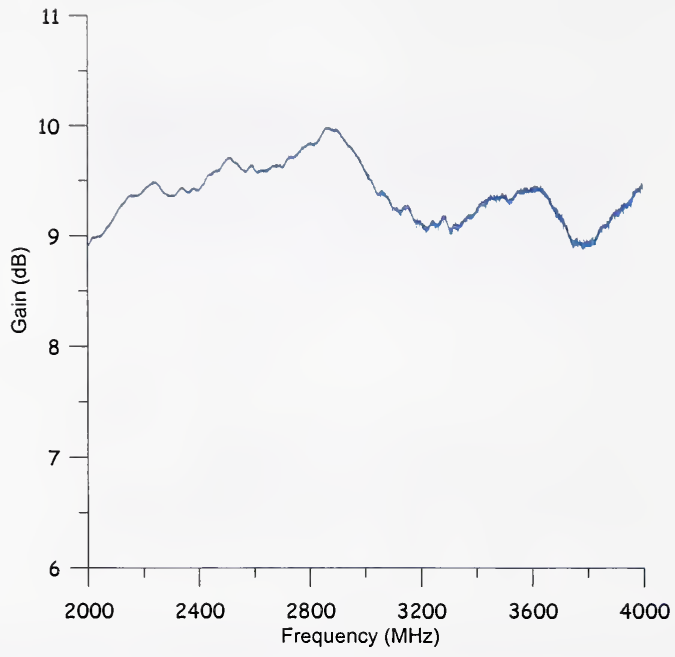

Figure 16. Gain measurement of double-ridged antenna, serial no. 3167.

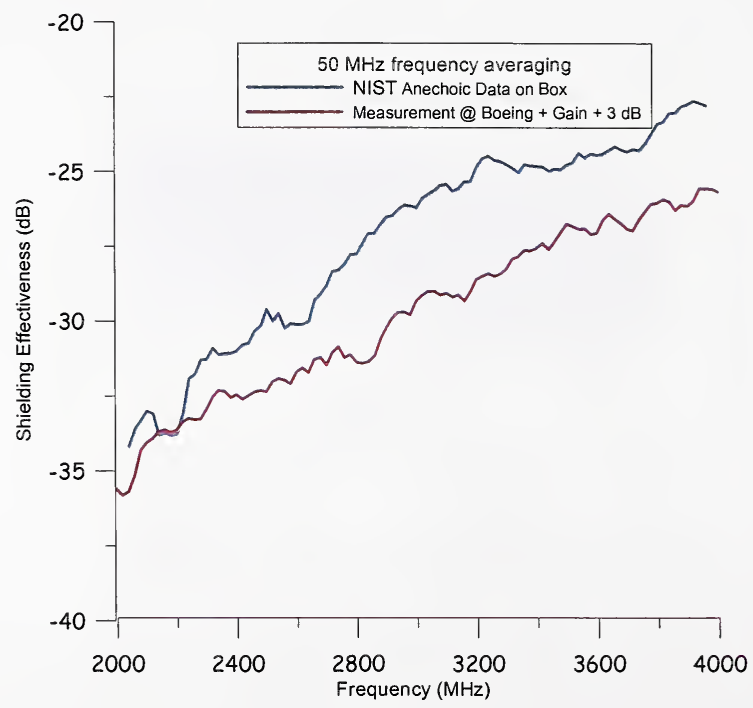

Figure 17. Comparison between anechoic data taken at NIST and final measurement data of box at aircraft hangar. 


\section{PHASE II: AIRCRAFT MEASUREMENTS}

The second phase consisted of an extensive series of shielding measurements performed on a Boeing 737-800 jet. The measurement program was carried out in the same hangar facility as the reference box measurements. Figure 18 shows the aircraft location inside the hangar and the various receive antenna locations. The aircraft measurements were much more demanding due to a greatly increased size and volume of the test artifact. The combination of large aircraft size, volume, and limited hangar space also limited the number of available measurement configurations. In addition, the large antenna separations made it necessary to use an opticallycoupled direct-pulse time-domain system instead of the VNA based system used for the box. Long cable lengths would have made it extremely difficult to maintain adequate phase stability, to maintain a wide dynamic range, and to achieve acceptable measurement fidelity. The measurement process was essentially unchanged with both the reference and penetration measurements being performed in the hangar. The use of a direct-pulse measurement system minimizes signal loss, increases dynamic range, and improves phase stability. The direct-pulse system is straightforward to implement with commercially available off-the-shelf hardware and standard instrumentation. The data acquired are amenable to digital signal-processing techniques to improve signal-to-noise ratios and reduce environmental effects.

Prior to the measurements at Boeing, we performed an extensive series of measurements on an all-metal iso-container located at the NIST facility in Boulder (see figure 19). The iso-container is an all-metal, box-like storage container that exhibits shielding characteristics quite similar to an actual aircraft. Since the observed signal levels obtained were similar to those of an aircraft, we used this initial set of measurements to optimize our hardware, signal processing methods, and fine-tune the measurement procedures. Due to time constraints, we had to implement an efficient testing sequence that provided a comprehensive shielding performance data set that characterized the key aircraft compartments. The aircraft compartments measured were: the center and first class positions of the passenger deck, the cockpit, the cargo bay (where luggage is stored), and the avionics bay, which contains key control, navigation and safety electronics circuitry.

\subsection{Measurement System for Aircraft Measurements}

The direct-pulse time-domain system is designed with separate transmitting and receiving units, and is configured to perform transmission measurements. The measurement system, depicted in figures 20 and 21, consisted of a pulse generator, three NIST-developed TEM hom antennas, 50-ohm coaxial interconnecting cables, and a digitizing sampling oscilloscope with a $12 \mathrm{GHz}$ bandwidth. For this measurement effort, the pulse generator operated at a $1 \mathrm{MHz}$ repetition rate, and triggering was provided by means of a low-loss high-fidelity, optical triggering link. The receiving system was located outside of the aircraft, and consisted of two TEM horns mounted at a $3.9 \mathrm{~m}$ height on a moveable tower outside of the aircraft. The sampling oscilloscope and a laptop computer were located at the base of the tower for data acquisition. We selected a $3.9 \mathrm{~m}$ height to place the antenna aperture centers at the same height as the passenger and cockpit windows. The receiving horns were oriented and aligned along a common 
axis with one horn oriented for horizontal polarization and the other for vertical polarization. The antenna outputs were fed into two-stage, low-noise broadband amplifiers, providing signal conditioning for the two-channel oscilloscope. This arrangement permitted the simultaneous reception of vertical and horizontal polarizations, with the immediate benefit of doubling measurement efficiency, thereby reducing the data acquisition time required in half-this increase in measurement efficiency is of paramount importance in time-critical efforts. The transmitter and its antenna were placed at selected locations inside of the aircraft. The reasons for this were threefold: (1) to take advantage of airframe shielding and reduce emissions leaking into the airport environment-this provided an additional safety margin against potential interference to airport communications, safety, and navigation systems that were located in close proximity to the measurement setup. An additional margin of safety was provided to the aircraft avionics systems by the use of a low-voltage, ultra-wideband pulse generator. (2) to permit measurements on an empty aircraft, and eliminate potential data variations due to measurement personnel, and (3) the transmitting configuration was far more compact, and could be deployed in some of the very tight aircraft compartments that could not accommodate either measurement personnel or the receiving equipment. For the larger volume aircraft compartments (passenger deck, cargo bay), a NIST-developed and fabricated $1.2 \mathrm{~m}$ TEM horn antenna was used. A more compact NIST $30 \mathrm{~cm}$ horn was used in the two smaller compartments: the cockpit and the avionics bay. The large TEM horns yield a flat amplitude spectrum and a phase-linear response over the one and one-half decade bandwidth of $30-1000 \mathrm{MHz}$, which is lower in frequency than the dual-ridged antennas used in the first phase. The fiber-optic trigger link was routed through a custom-manufactured test hatch, located on the bottom of the fuselage directly ahead of the nose wheel. This test hatch is used in current certification tests by the manufacturer for field testing of aircraft.

The measurement setup used to obtain shielding performance data is depicted in figure 22 . The process was straightforward and consisted of two steps: (1) a reference transmission measurement, shown in figure 23, was first taken with the TEM horn antennas located in an unobstructed portion of the hangar, and (2) the transmitting antenna was then placed in a selected aircraft compartment, and a second transmission measurement through the airframe was performed. In the reference measurement, the two receiving antennas were mounted on the same $3.9 \mathrm{~m}$ tower used for the aircraft measurements; the transmitting antenna was mounted on another identical tower. The antennas were then aligned along a common boresight axis at a separation of $4.24 \mathrm{~m}$. This distance was chosen to permit efficient isolation of the direct antennato-antenna coupling and to minimize the effects of environmental scattering.

Shielding performance was obtained from the reference and penetration waveforms using the signal-processing sequence shown in figure 24. The reference waveform is first time-gated to isolate the direct antenna-to-antenna coupling and to suppress interference from environmental scattering. The resulting waveform, shown in figure 25 , is a virtual free-space reference. A Fourier transform is then applied to the gated waveform to obtain a free-space reference amplitude spectrum. This spectrum is mathematically equivalent to that obtained with boresighted antennas in a completely unobstructed free-space environment of infinite extent. Boresighted TEM horn antennas with a separation greater than $0.25 \mathrm{~m}$ exhibit an amplitude 
spectrum that drops $6 \mathrm{~dB}$ as the distance is doubled, and exhibit a well-defined $1 / \mathrm{r}$ decay in signal as the aperture-to-aperture separation, $\mathrm{r}$, is increased. In the penetration measurements, the measurement distance was defined from the aperture center of the receiving horn to the geometric center of the aircraft, as shown in figure 2.6. In order to account properly for this, the following correction factor in $\mathrm{dB}$ is subtracted from the time-gated reference amplitude spectrum:

$$
F_{c}(d B)=20 \log _{10}\left(\frac{D}{D_{r}}\right),
$$

where $D_{\mathrm{r}}=4.24 \mathrm{~m}$, the reference distance, and $\mathrm{D}$ is the horizontal distance from the aperture center of the receiving antenna to the geometric center of the aircraft. Using the aircraft geometric center as a reference point is more accurate as the measurement distance increases. This methodology works well for outdoor measurement scenarios, where the receiving antenna can be placed at.large distances (typically $30 \mathrm{~m}$ or more) [4]. In the hangar environment, the permitted measurement distances were more restricted, ranging from $6.7 \mathrm{~m}$ to $20.4 \mathrm{~m}$, thereby introducing potential systematic errors into the shielding results. The amount of error introduced at closer distances is a function of the location and distribution of the leakage radiation from the aircraft, with the most accurate results obtained with a radiating center of infinitesimal extent at the geometric center of the aircraft. A description of detailed leakage mechanisms is beyond the scope of this effort, but is, however, a current topic of research at NIST. The Reverberant Reference Method, discussed in section 3.8 was developed during the course of this effort to overcome these potential limitations.

\subsection{Extrapolation Measurements}

The initial series of measurements consisted of placing the transmitting antenna in the main passenger cabin, with the antenna aperture over the geometric center of the aircraft. The receiving TEM horns were positioned at various locations along the so-called extrapolation line depicted in figure 26. This line permitted the widest range of antenna positions and the largest standoff distance from the aircraft. The receiving antennas were positioned at a 45 -degree angle $\left(\theta=-135^{\circ}\right)$ from the centerline of the aircraft, and boresighted at the aircraft center. Measurements were taken at distances of $23.4 \mathrm{~m}, 20.4 \mathrm{~m}, 17.4 \mathrm{~m}, 14.3 \mathrm{~m}, 11.3 \mathrm{~m}, 8.2 \mathrm{~m}$, and $6.7 \mathrm{~m}$. Figures 27 and 28 show plots of shielding performance data obtained using a fixed reference distance of $4.24 \mathrm{~m}$ and an averaging bandwidth of $50 \mathrm{MHz}$. Data are shown at each location along the extrapolation line for horizontal and vertical polarization. The spread in shielding data is greater than $10 \mathrm{~dB}$ for frequencies above $200 \mathrm{MHz}$. Once we applied the distance correction of equation 5 , as discussed above, the data variations were significantly reduced, as is seen in figures 29 and 30. The results in figures 29 and 30 indicate that the leakage from the aircraft is governed by an inverse distance law $(1 / \mathrm{r})$; the receiving antennas were in the far-field of the radiating antenna. Such a trend is physically reasonable, if the leakage radiation is assumed to be localized around the aircraft center-such a scenario could occur if the passenger cabin windows were radiating in the immediate vicinity of the transmitting antenna. The shielding exhibits values ranging from $30 \mathrm{~dB}$ to less than $0 \mathrm{~dB}$ at $290 \mathrm{MHz}$. Maximum shielding values were obtained below $200 \mathrm{MHz}$. In the $200-300 \mathrm{MHz}$ frequency range, the shielding drops off rapidly until minimum 
performance was obtained around $300 \mathrm{MHz}$. In fact, with the antenna placed at $20.4 \mathrm{~m}$, no shielding performance was realized for horizontal polarization, and slightly negative shielding was realized at $300 \mathrm{MHz}$. This high penetration might be due to a combination of aperture array effects and possible aircraft-hangar interactions. Obtaining negative values of shielding is not unreasonable, recent numerical studies conducted at NIST (Boulder) under the sponsorship of the FAA have shown that negative shielding results can be obtained on canonical shielding structures - these studies have attributed this phenomenon to our definition of shielding performance in which we use a free-field reference as a basis of comparison. Above $300 \mathrm{MHz}$, the shielding increased once again, with values ranging from $10-15 \mathrm{~dB}$ above $500 \mathrm{MHz}$.

To increase the signal-to-noise ratio, we can use time-domain gating to remove the portion of the time-domain record where the signal fades into the noise. This process is illustrated in figures 31-33. Figure 31 contains a time-domain plot of the amplitude-squared record obtained at the $26.5 \mathrm{~m}$ extrapolation position. Included in the plot (red line) is a least-mean-squares linear fit to the data; and if we extrapolate down in time, we can see that the signal disappears into the noise at approximately $600 \mathrm{~ns}$. This suggests applying a 0-600 ns gate, in which the time-domain record is forced to zero from $600 \mathrm{~ns}$ to the end of the record. The improvement achieved using a time gate is seen by comparing figures 32 and 33 . By gating out system noise we can increase the signal-to-noise ratio by approximately $5 \mathrm{~dB}$ and also increases dynamic range.

\subsection{Passenger Deck (Center) Measurements}

The next sequence of measurements was conducted with the transmitting antenna's location the same as that for the extrapolation measurements. In this case, the receiving antenna was moved to a number of different positions shown in figure 34: (1) the tail position, $24.4 \mathrm{~m}$ from transmit antenna, (2) the wing position, $21.3 \mathrm{~m}$ from transmit antenna, (3) the forward position, $14.6 \mathrm{~m}$ from transmit location at an azimuthal angle of 42 degrees from the centerline of the aircraft, (4) two nose positions at $26.7 \mathrm{~m}$ and $30.5 \mathrm{~m}$, and finally, (5) the opposite position, $14.6 \mathrm{~m}$ from the transmit location at an angle of 42 degrees from the centerline of the aircraft but exactly opposite from the forward position on the same side of the aircraft as the cargo-bay door. The shielding performance data obtained are shown in figures 35 and 36 for which a distance correction has been applied along with the averaging bandwidth of $50 \mathrm{MHz}$. It is interesting to note that the general frequency dependence is quite similar to that obtained in the extrapolation measurements-independent of position, with minimum shielding values once again occurring in the vicinity of $300 \mathrm{MHz}$. Clearly the location of the transmitting antenna has a strong influence on the general shielding trends. Below the vicinity of $300 \mathrm{MHz}$, the shielding performance rapidly increases to a maximum value and, and above this frequency it also increases, but more gradually. The lowest shielding performance was measured at the tail and wing positions for most of the frequency band shown in the figures. If we power-average the horizontally polarized data over all of the positions with the frequency fixed at $500 \mathrm{MHz}$, we obtain an average shielding of $17 \mathrm{~dB}$, with an associated spread of approximately $5 \mathrm{~dB}$. Similarly, for the vertically polarized data, the average shielding is $14 \mathrm{~dB}$ with a somewhat larger spread of $8 \mathrm{~dB}$. 


\subsection{Passenger Deck (First Class) Measurements}

We moved the transmit antenna to the forward part of the passenger deck, where the firstclass cabin is located. Shielding data is shown in figures 37 and 38 for horizontal and vertical polarization, respectively. The trends are somewhat different than previously encountered-the shielding increases to a maximum value below $400 \mathrm{MHz}$ and rapidly drops below $100 \mathrm{MHz}$. The new transmitting antenna location shifted the frequency response. Also, it is interesting that the shielding performance at the nose positions was lower than that for the tail and extrapolation positions by approximately $10 \mathrm{~dB}$. At $500 \mathrm{MHz}$, the average shielding performance for horizontal polarization was $10 \mathrm{~dB}$ with excursions from 5 to $15 \mathrm{~dB}$. The average shielding performance for vertical polarization was approximately $12 \mathrm{~dB}$ with excursions from 5 to $17 \mathrm{~dB}$. The results obtained are quite reasonable: the transmitting antenna was closer to the cockpit windows so the radiated fields could couple to more windows. We therefore encounter lower shielding values at the nose positions and the forward and opposite positions.

\subsection{Cockpit Measurements}

A series of shielding measurements were performed with the transmitting horn located in the cockpit. The tight space encountered in the cockpit necessitated the use of a smaller $30 \mathrm{~cm}$ TEM horn antenna. In this test setup, depicted in figure 39, the antenna was boresighted at the instrumentation directly above the cockpit windows. Shielding performance results are depicted in figures 40 and 41 for horizontal and vertical polarization respectively. Immediately apparent is the radically different shielding results from those obtained on the passenger deck. The confined space of the cockpit and the close proximity of the windows exert a strong influence on the results obtained. The data obtained with the receiving antennas placed at the two nose positions exhibited dramatically lower shielding numbers than those obtained at the other positions. This is not surprising since the transmitting antenna was quite close to the larger cockpit front windows, and we expect larger amounts of radiation in the direction of the nose. Another major difference is the absence of a pronounced resonant dip in shielding that occurred in the passenger deck measurements - this is probably due to the presence of larger radiating apertures in the cockpit. At a frequency of $500 \mathrm{MHz}$, the average shielding values were $9 \mathrm{~dB}$ for the nose positions, with a spread of $3 \mathrm{~dB}$, and for the other positions we saw an average shielding performance of $20 \mathrm{~dB}$ with a spread of $5 \mathrm{~dB}$.

\subsection{Cargo Bay Measurements}

The transmitter was next deployed in the cargo bay and the measurement sequence was carried out once again. Because of the large volume available, a $1.2 \mathrm{~m}$ TEM hom was used. The initial data indicated that the compartment was electrically "tight." The difference in signal levels is depicted in figure 42 , where time-domain records obtained in the passenger deck and cargo bay can be directly compared. The graph highlights the difference in received signal levels between a measurement taken along the extrapolation line at $6.71 \mathrm{~m}$ and a measurement taken from the cargo bay in the "special test" position, depicted in figure 43. The passenger deck signal was much larger than that of that of the cargo bay. Not only that, the buildup of the passenger deck 
signal was rapid, whereas the cargo bay signal exhibits a much slower buildup. Due to the weak signal levels encountered with the receiving antennas boresighted with geometric center of the aircraft, we developed a "special test" position, in which we re-boresighted the receiving antenna directly at the cargo bay door, to maximize received signal levels. This test indicated that radiation was emanating from the seams in the cargo door. From figures 44 and 45 , we see that the shielding performance for the "special test" position is still quite high, and decreases above $600 \mathrm{MHz}$ for horizontally polarized components, but remains virtually the same for vertically

polarized components. The shielding performance values for the cargo bay ranged from $20 \mathrm{~dB}$ to $30 \mathrm{~dB}$, at $500 \mathrm{MHz}$, with an average value of $25 \mathrm{~dB}$.

\subsection{Avionics Bay Measurements}

The final compartment to be characterized was the avionics bay, which contains key circuitry for the control, safety, and navigation systems on the aircraft. The avionics bay also has the smallest volume of all the compartments tested, which required the use of the $30 \mathrm{~cm}$ TEM horn antenna. A visual inspection of the avionics bay revealed a number of open apertures to the adjacent cargo bay, so we realized that the avionics bay could couple strongly to the cargo bay. Thus, once again, we used our "special test" setup (figure 43) with the receiving antennas boresighted at the cargo bay door. Distance-corrected shielding performance measurements are shown in figures 46 and 47 . We note that the "special test" setup again exhibited the lowest measured shielding performance, and the nose positions yielded the second lowest shielding performance. A visual inspection conducted inside the avionics bay indicated possible coupling paths into the cockpit which could account for the enhanced radiation from the nose of the aircraft. The tail and extrapolation position had the highest shielding performance values. The average shielding performance for this compartment was around $18 \mathrm{~dB}$, at $500 \mathrm{MHz}$, with a spread in the data of $\pm 5 \mathrm{~dB}$.

\subsection{Comparison of Different Compartments}

We also looked at the difference between various shielding performance values of the compartments at a fixed receiving location. This is shown in figure 48 , in which the five transmitting positions are plotted at both $12 \mathrm{~m}$ and $23.5 \mathrm{~m}$ along the extrapolation line. We chose to plot the data at these two distances because we did not have a complete data set for each transmit position at both distances. At these locations, the cargo bay showed the highest shielding performance, at approximately $30 \mathrm{~dB}$. The passenger deck and first class cabin showed the lowest shielding performance with values ranging from 10 to $15 \mathrm{~dB}$. The avionics bay initially exhibited high shielding, similar to the cargo bay. The shielding rapidly decreased in the $200-400 \mathrm{MHz}$ frequency band, and exhibited characteristics within 1-2 dB of the cockpit above $400 \mathrm{MHz}$. This indicated strong coupling between the avionics bay and the cockpit, which was also postulated in Section 3.7.

\subsection{Summary of Direct Inumination Aircraft Measurements}

In order to concisely summarize shielding performance, the results were linearly averaged 
as a function of the receiving location. A summary of the averaged shielding performance for combinations of transmitting and receiving locations is given in Table 1 for $500 \mathrm{MHz}$. The cargo bay had the highest shielding performance with an average value of $26 \mathrm{~dB} \pm 5 \mathrm{~dB}$. The other compartments had approximately the same shielding performance, with an average value of 14 $\mathrm{dB} \pm 5 \mathrm{~dB}$. The cockpit had the lowest shielding performance at the two nose positions, with an average value of $9 \mathrm{~dB} \pm 6 \mathrm{~dB}$. This is logical because the cockpit windows provide the largest radiating apertures in that portion of the aircraft. The highest shielding performance for the cockpit occurred at the tail position with a value of $20 \mathrm{~dB}$. For the avionics bay, the highest shielding values occurred at the extrapolation and tail positions, with values of $22 \mathrm{~dB}$ and $17 \mathrm{~dB}$, respectively. This is reasonable since these two positions were furthest from the transmit location and there are no direct signal paths.

The measured data for the direct illumination technique showed reasonable average shielding performance values for the aircraft. The measurement method lends itself well to either measurements inside or outside the hangar environment. Measurements performed outside the hangar naturally minimize possible reflective sources. Reflective sources within the hangar can be removed and the signal enhanced using digital signal-processing techniques. The instrumentation used is simple to incorporate and less expensive to purchase and maintain than conventional spectrum analyzer based instrumentation.

Table 1. Average shielding performance (in $\mathrm{dB}$ ), at $500 \mathrm{MHz}$, for various transmitting and receiving locations with an averaging bandwidth of $50 \mathrm{MHz}$.

\begin{tabular}{c|ccccccc} 
& Tail & $\begin{array}{c}\text { Extrapo- } \\
\text { lation }\end{array}$ & Wing & Forward & $\begin{array}{r}\text { Nose@ } \\
23.6 m\end{array}$ & $\begin{array}{r}\text { Nose@ } \\
26.7 m\end{array}$ & Opposite \\
\hline $\begin{array}{c}\text { Passenger } \\
\text { Deck } \\
\text { (Center) }\end{array}$ & 15 & - & 16 & 17 & 17 & 17 & 17 \\
\hline $\begin{array}{c}\text { Passenger } \\
\text { Deck 2 } \\
\text { (First class) }\end{array}$ & 18 & 18 & 16 & 17 & 14 & 14 & 19 \\
\hline Cockpit & 20 & 19 & 15 & 15 & 9 & 9 & 18 \\
\hline Cargo Bay & 27 & 30 & 25 & 27 & 23 & 24 & 25 \\
\hline Avionics Bay & 17 & 22 & 15 & 15 & 14 & 14 & 14
\end{tabular}

\subsection{The Reverberant Reference Method}

During the course of this effort, a new method for obtaining a baseline reference measurement was developed. This method is called the Reverberant Reference Method, and it provides an alternative technique for obtaining a reference signal. It is designed to be used for shielding measurements in closed, reverberant environments, such as an all-metal aircraft hangar. This technique originated during the data processing of the phase I box measurements. During 
the phase I box measurements, we performed antenna-to-antenna measurements for a number of testing configurations in the hangar environment to quantify the amount of reverberant behavior. During the course of our data processing, we discovered that if the hangar reverberation was used as a reference for the box measurements, in place of the boresighted antenna-to-antenna reference, the correction factors for the gain of the antenna and the polarization mismatch were not necessary to obtain agreement, to within $3 \mathrm{~dB}$, with those obtained using reverberationchamber techniques (we have not shown these results in this report due to the limited amount of data obtained and unknown uncertainties in the early box data).

As a result of the preliminary box results obtained, we incorporated the reverberant reference method into our phase II aircraft shielding test plan. Figure 49 illustrates the reverberant reference test setup. The antennas were deployed in the hangar environment at a larger separation of $26.1 \mathrm{~m}$ in order to enhance hangar reverberation, and to reduce the effects of direct antenna-to-antenna coupling. Additional suppression of the direct coupling component was achieved by using the aircraft as a blocking obstacle and boresighting the antennas at different angles. This configuration was optimal for capturing the reverberant effects of the hangar. Figure 50 shows the time-domain waveform of the received reverberant field obtained with this test configuration. The ratio between peak signal levels between the direct antenna reference (see figure 25) and the reverberant reference (see figure 50) is approximately 100. Although the reverberant reference has a greatly reduced amplitude, it has a much greater temporal extent; resulting in a similar signal energy level to that of the direct reference. The signal processing sequence used to obtain shielding with the reverberant reference is shown in figure 51. The data processing used here differs from that of figure 24 in two respects: no distance correction is needed and, the reference is frequency-averaged over the same bandwidth as that of the penetration signal. Depending on the orientation of the antennas in the reference test setup, it might be necessary to time gate the waveform to suppress the direct antenna-toantenna coupling. This method of obtaining a reference must emphasize hangar reverberation, and direct antenna coupling must be suppressed to obtain correct results. A comparison of shielding results obtained using the reverberant and direct antenna-to antenna references are shown in figures 52-54, for various transmit and receive locations. The differences in the results obtained are quite interesting. First, the results obtained using the reverberant hangar reference and the direct reference exhibit very similar frequency-response trends above $150 \mathrm{MHz}$, and the results obtained agree well in most instances. One interesting result is seen in figure 52 for the case of the transmitter in the first-class passenger deck and the receiving antenna located near the nose of the aircraft. The direct antenna-to-antenna reference obtained a low shielding performance of near $0 \mathrm{~dB}$ at $440 \mathrm{MHz}$, whereas, the reverberant reference yielded a result that is $7-8 \mathrm{~dB}$ higher-this eliminates the zero- or negative-gain shielding anomaly. One surprise was the similarity of results obtained using the two methods. This is still a topic of research in our project, but we can speculate as to why the two methods give similar results. We believe that if the TEM antennas had large, directive gain, then the difference between the two methods would be the gain of the antenna. However, since these TEM antennas have low directive gain, the two techniques yield comparable results. 


\subsection{Uncertainty Analysis}

A general uncertainty analysis for measurements performed for the box and airplane is given in tables 2 through 4 [10]. Type- $A$ and Type- $B$ uncertainties are combined to yield an overall combined uncertainty with a coverage factor of $\mathrm{k}=2$. The first table summarizes the contributions to uncertainty for the reference measurements, and the second table summarizes contributions for the box penetration measurements and the last table summarizes contributions for the aircraft penetration measurements. The type- $A$ uncertainties based on repeated measurements use a small sample size because time limitations did not permit an extensive set of repeated measurements. Antenna position uncertainties for the reference were determined using the maximum possible deviation in distance between the antennas. To determine the distance correction uncertainty, we use eq. (5) and a Monte-Carlo simulation. Drift uncertainties are due to the instrumentation and were determined from an extensive set of laboratory measurements here at NIST. Antenna polarization mismatch errors were determined using a series of extrapolation measurements in the NIST time-domain free-field laboratory. The signal-to-noise ratios for both the reference and the aircraft were inferred from system noise floor measurements shown in figures 32 and 33 . In the reference measurement, the primary contributors to measurement uncertainty are the system Type-A repeatability, time gating, and system noise.

Table 2. Reference antenna-to-antenna uncertainties $(\mathrm{dB})$ with a coverage factor of $\mathrm{k}=2$.

\begin{tabular}{c|ccc}
\hline & $200 \mathrm{MHz}$ & $500 \mathrm{MHz}$ & $800 \mathrm{MHz}$ \\
\hline Measurement Repeatability & \pm 0.5 & \pm 0.4 & \pm 0.3 \\
Range Uncertainties / Distance Correction & \pm 0.04 & \pm 0.04 & \pm 0.04 \\
Time Gating & \pm 0.3 & \pm 0.2 & \pm 0.1 \\
Drift & \pm 0.1 & \pm 0.2 & \pm 0.2 \\
Polarization Mismatch & \pm 0.1 & \pm 0.1 & \pm 0.1 \\
SNR & \pm 0.2 & $\mathrm{Neg}$ & \pm 0.3 \\
\hline Combined RSS Uncertainty & \pm 0.6 & \pm 0.5 & \pm 0.5 \\
\hline
\end{tabular}

Table 3. Shielding Performance uncertainties (dB) for Box

\begin{tabular}{c|ccc}
\hline & $2000 \mathrm{MHz}$ & $3000 \mathrm{MHz}$ & $4000 \mathrm{MHz}$ \\
\hline Measurement Repeatability & \pm 0.5 & \pm 0.4 & \pm 0.3 \\
Distance Correction / Near Field Effects & \pm 1.0 & \pm 1.0 & \pm 1.0 \\
Drift & \pm 0.1 & \pm 0.2 & \pm 0.2 \\
Time Gating & $\mathrm{Neg}$ & $\mathrm{Neg}$ & $\mathrm{Neg}$ \\
Polarization Mismatch & \pm 0.1 & \pm 0.1 & \pm 0.1 \\
SNR & \pm 0.1 & \pm 0.1 & $\mathrm{Neg}$ \\
Antenna-to-Antenna Reference & \pm 0.6 & \pm 0.5 & \pm 0.5 \\
(From Table 2) & & & \\
\hline Total Combimed RSS Uncertainty (dB) & \pm 1.3 & \pm 1.2 & \pm 1.2 \\
for Box Shielding & & & \\
\hline
\end{tabular}


Table 4. Shielding Performance uncertainties $(d B)$ for aircraft.

\begin{tabular}{c|ccc}
\hline & $200 \mathrm{MHz}$ & $500 \mathrm{MHz}$ & $800 \mathrm{MHz}$ \\
\hline Measurement Repeatability & \pm 0.7 & \pm 0.6 & \pm 0.5 \\
Distance Correction / Near Field Effects & \pm 1.4 & \pm 1.4 & \pm 1.4 \\
Drift & \pm 0.1 & \pm 0.2 & \pm 0.2 \\
Time Gating & $\mathrm{Neg}$ & $\mathrm{Neg}$ & $\mathrm{Neg}$ \\
Polarization Mismatch & \pm 0.1 & \pm 0.1 & \pm 0.1 \\
SNR & \pm 0.8 & \pm 1.0 & \pm 0.7 \\
Antenna-to-Antenna Reference & \pm 0.6 & \pm 0.5 & \pm 0.5 \\
(From Table 2) & & & \\
\hline Total Combimed RSS Uncertainty (dB) & \pm 1.9 & \pm 1.9 & \pm 1.7 \\
for Aircraft Shielding & & & \\
\hline
\end{tabular}

Measurement repeatability, distance correction, system noise, and the antenna-to antenna reference are the primary contributors to the uncertainty in the shielding performance measurement. Due to the lower signal levels encountered in the penetration measurement, the shielding performance exhibits significantly higher uncertainties than those encountered in the reference measurements.

\subsection{Conclusions for the Phase II Aircraft Effort}

The results obtained in this effort demonstrate the effectiveness and versatility of the NIST direct-pulse time-domain system for conducting in situ shielding measurements of a large commercial aircraft. We have demonstrated that the NIST-developed measurement system can effectively perform in a commercial manufacturing environment and generate shielding results for all of the major aircraft compartments. We have also demonstrated the directional capabilities of this system by placing the receiving antenna at a number of selected positions. In addition, the special test conducted with the transmitter inside the cargo bay demonstrates the ability of the NIST system to identify specific leakage points from the aircraft. We also developed a new technique, the reverberant reference method, which exploits environmental scattering. The reverberant reference method yields results that are similar to the direct antennato-antenna reference method, with the added bonus that some $0 \mathrm{~dB}$ and negative shielding anomalies are eliminated. The reasons why this occurs is a current topic of research at NIST. All in all, the shielding results obtained exhibit good fidelity and low uncertainties. The effort clearly demonstrates that the NIST-developed time-domain measurement system is an excellent tool because of its versatility, accuracy, and cost-effectiveness.

\section{ACKNOWLEDGEMENTS}

We thank Mike Hatfield and Bill Price for funding the effort and allowing us to develop our technical expertise in these highly critical measurements. We also thank Bill Daniels for providing his technical support and expertise in this area. From NIST, we thank Andy Ondrejka, John Ladbury, and Galen Koepke for laying the groundwork for these types of measurements and for 
providing valuable input. We also thank Perry Wilson, Dennis Friday, and Andy Repjar for their valuable contributions and support of this measurement effort.

\section{REFERENCES}

[1] Ladbury, J.M., Lehman, T.H., Koepke, G.H. Coupling to devices in electrically large cavities or Why classical EMC evaluation techniques are becoming obsolete. 2002 EMC International Symposium on EMC, 2002, August.

[2] Hill, D.A.; Ma, M.T.; Ondrejka, A.R.; Riddle, B.F.; Crawford, M.L.; Johnk, R.T. Aperture Excitation of Electrically Large, Lossy Cavities. Natl. Inst. Stand. Technol. Tech. Note $1361 ; 1993$ September.

[3] Hill, D.A.; Adams, J.W.; Ma, M.T.; Ondrejka, A.R.; Riddle, B.F.; Crawford, M.L.; Johnk, R.T. Aperture Excitation of Electrically Large, Lossy Cavities. IEEE Trans. Electromag. Compat., 36(3), 169-179, 1994 August.

[4] Freyer, G.J.; Hatfield, M.O.; Loughry, T.A.; Johnk, R.; Johnson, D.M, Ondrejka, A.R. Shielding effectiveness measurements for a large commercial aircraft. IEEE Int. Symp. Electromag. Compat., Atlanta, GA, 1995, August, 383-386.

[5] Johnson; M.; Hatfield, M.O.; Slocum, M.B.; Loughry, T. A.; Ondrejka, A.R.; Johnk, R.T.; Freyer, G.J. Phase II demonstration test of the electromagnetic reverberation characteristics of a large transport aircraft. Dahlgren Division, Naval Surface Warfare Center Tech. Note NSWCDD/TR-97/84, 1997 September.

[6] Ma, M.T.; Kanda, M. Electromagnetic compatibility and interference metrology. Natl. Inst. Stand. Technol. Tech. Note 1099; 1986 June.

[7] Ladbury, J.M.; Johnk, R.T.; Ondrejka, A.R. Rapid evaluation of mode-stirred chambers using impulsive waveforms. Natl. Inst. Stand. Technol. Tech. Note 1381; 1996 June.

[8] Hill, D.A. Electronic mode stirring for reverberation chambers. IEEE Trans. Electromag. Compat., 36(4), 294-299, 1994 November.

[9] Stutzman, W. L., Thiele, G.A. Antenna theory and design. John Wiley \& Sons; 1981.

[10] Novotny, D.R., Johnk, R.T., Ondrejka, A.R. Analyzing broadband, free-field, absorber measurements. 2001 EMC International Symposium on EMC, Vol. 2, 1152-1157, 2001, October. 


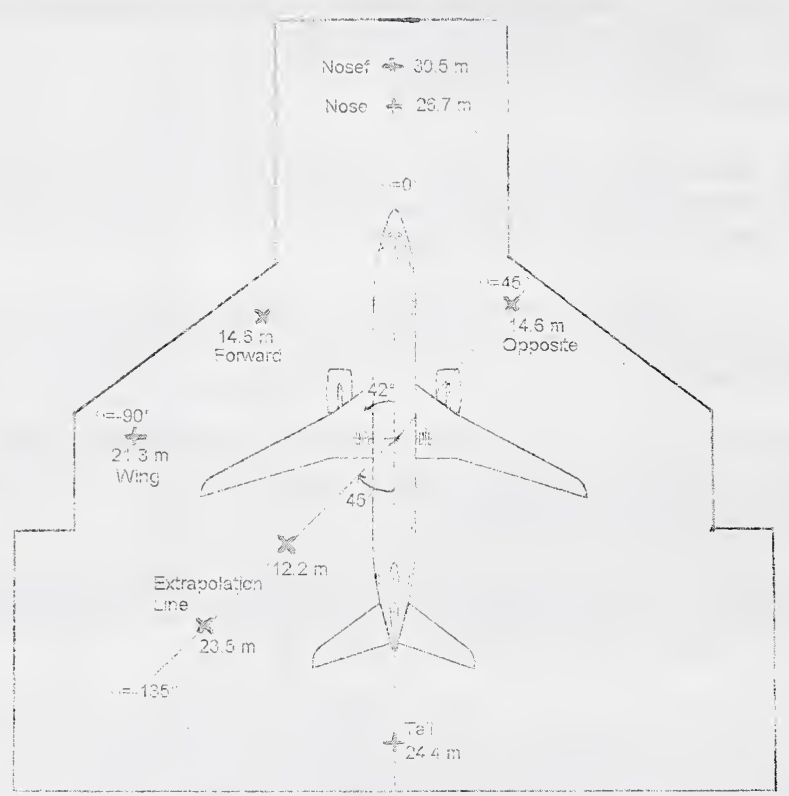

Figure 18. Diagram of aircraft in hangar.

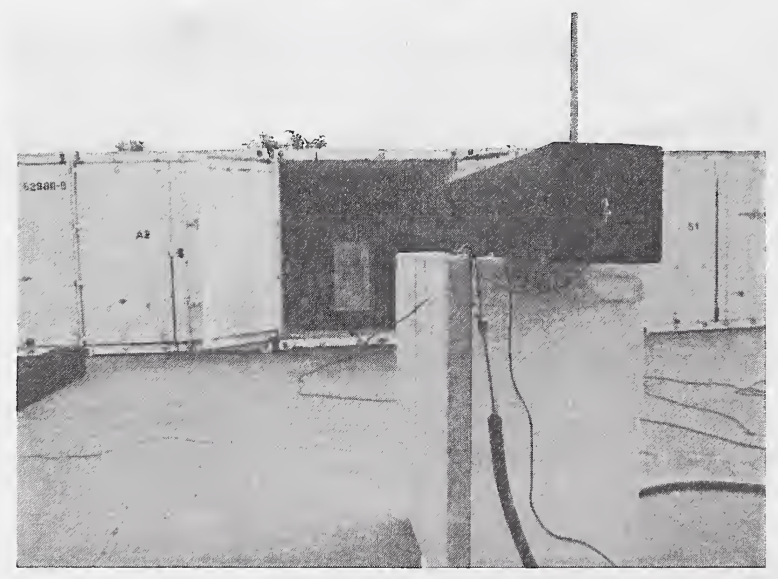

Figure 19. Antenna measurements in iso-container to simulate actual aircraft conditions. 


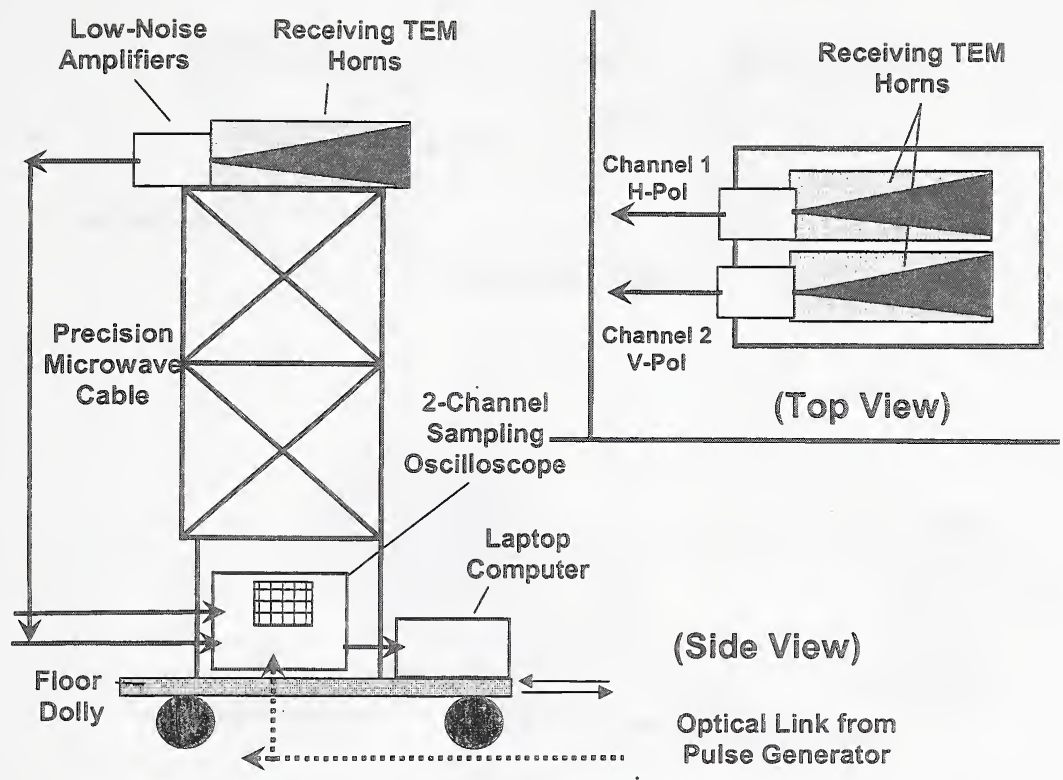

Figure 20. Measurement system for aircraft measurements.

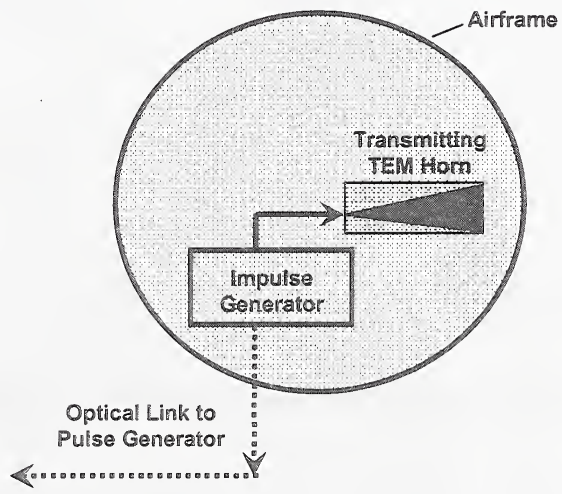

Figure 21. Transmitting equipment located within aircraft frame. 

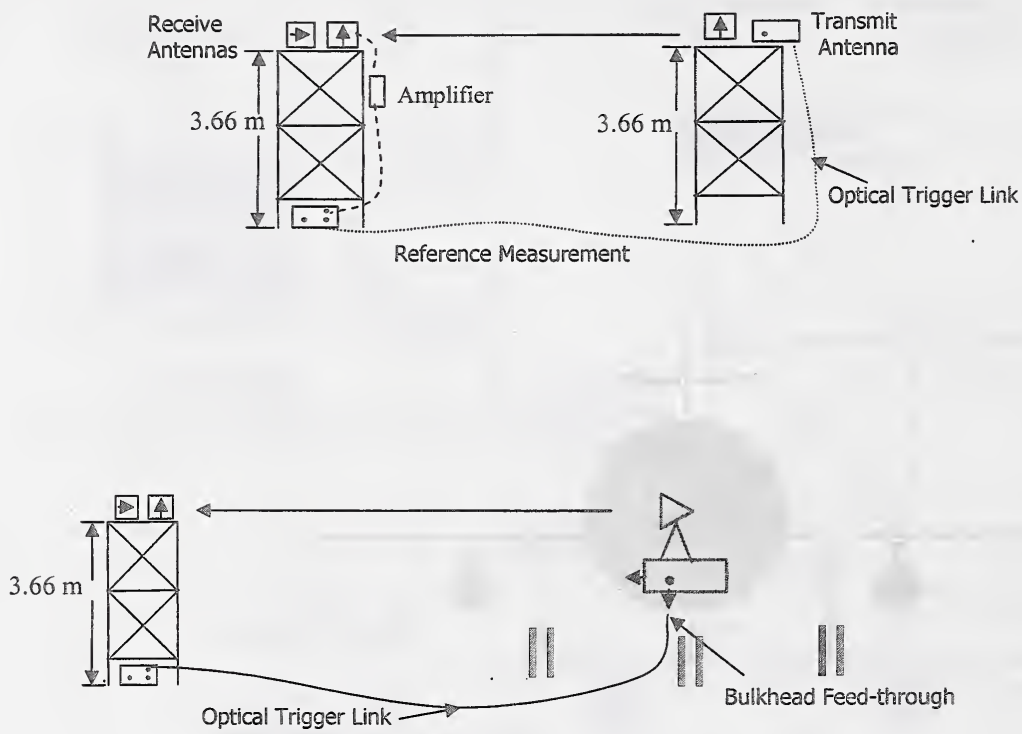

Figure 22. Measurement setup for reference and penetration measurements of aircraft. 


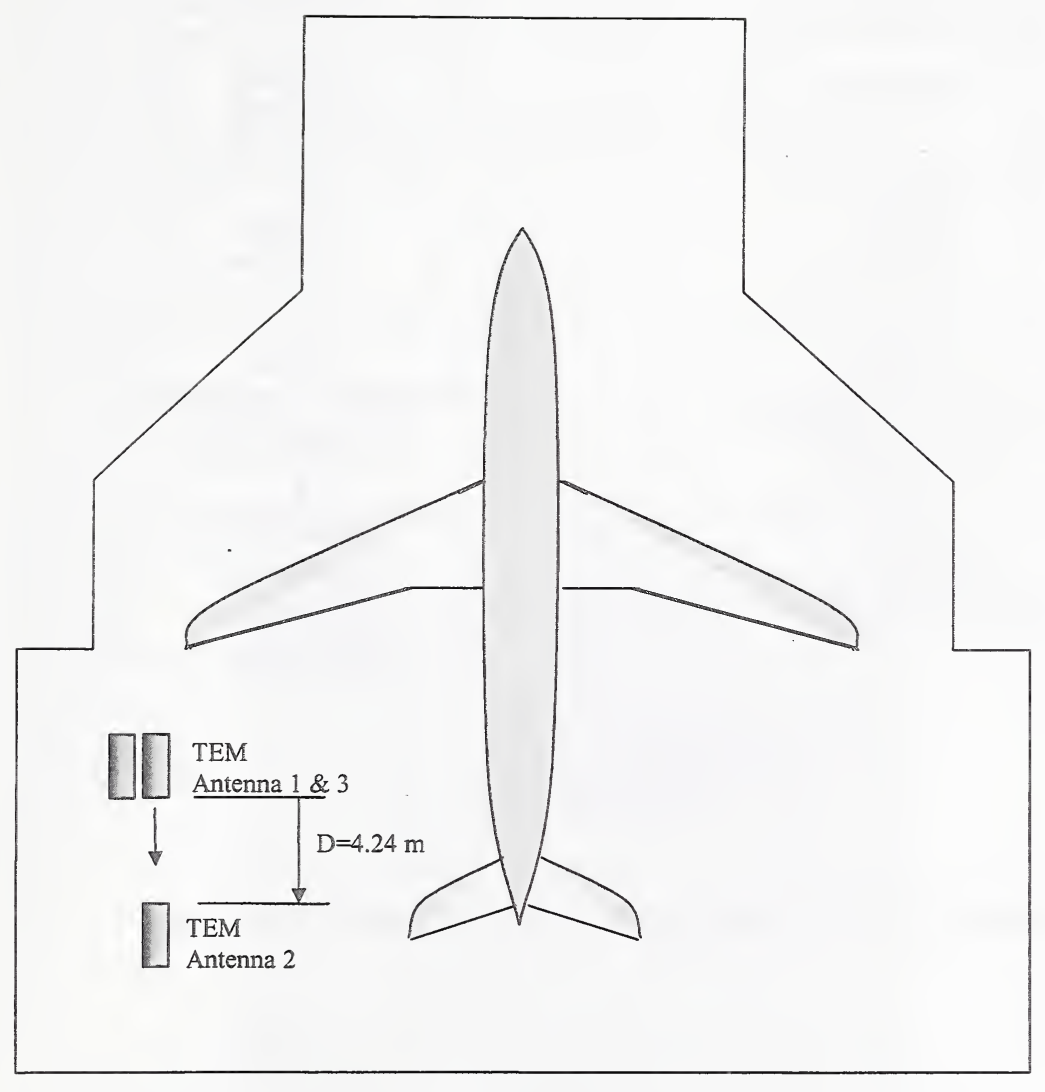

*Drawing not to scale

Figure 23. Reference measurement taken with aircraft in hangar. 


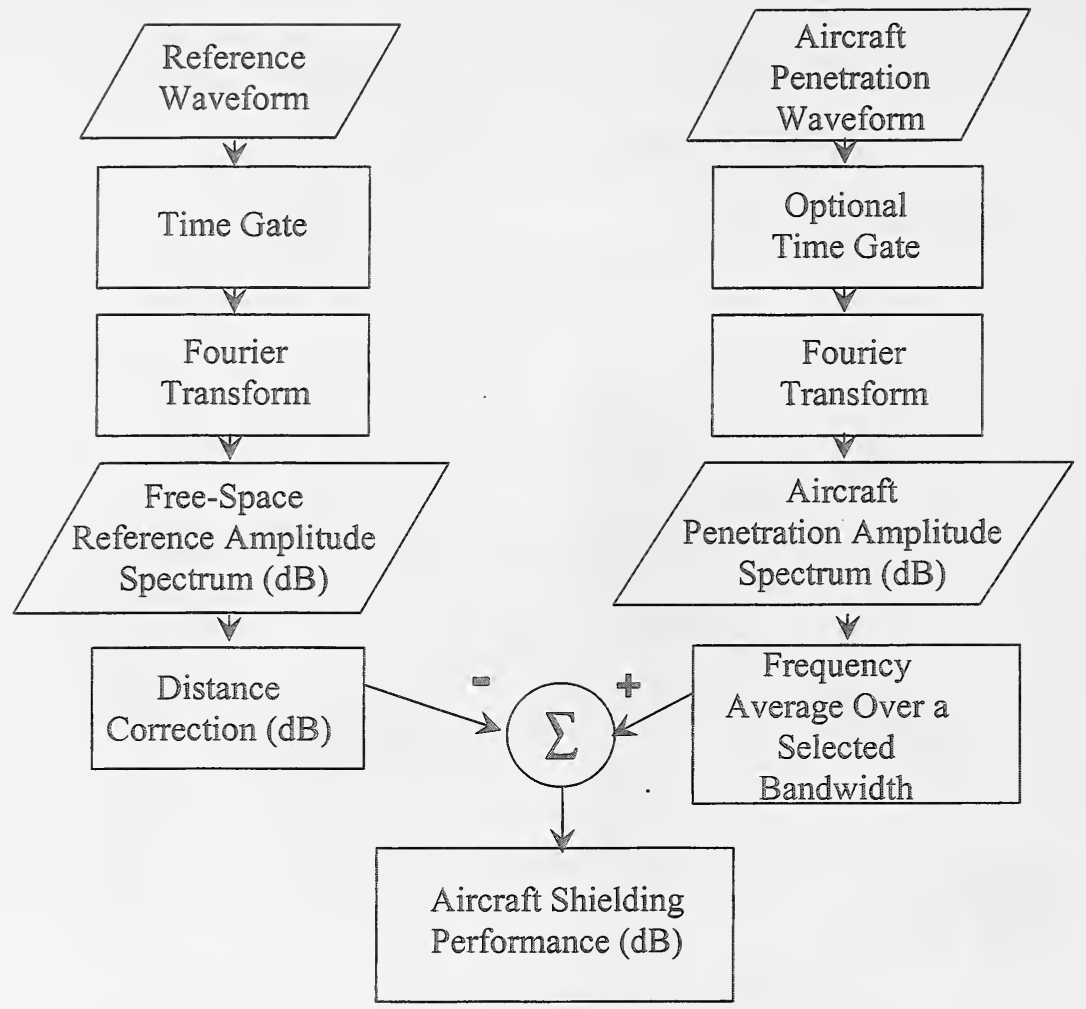

Figure 24. Signal processing sequence to obtain aircraft shielding performance. 


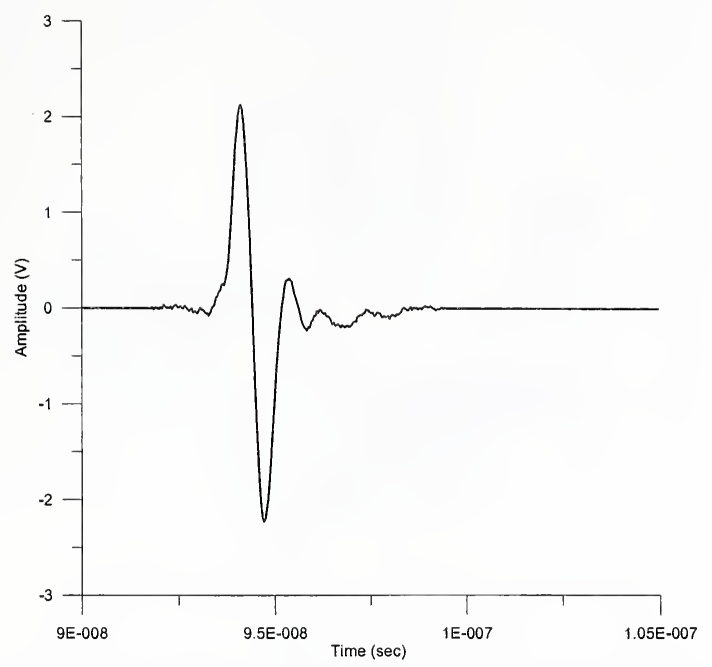

Figure 25. Reference measurement after time-domain gating.

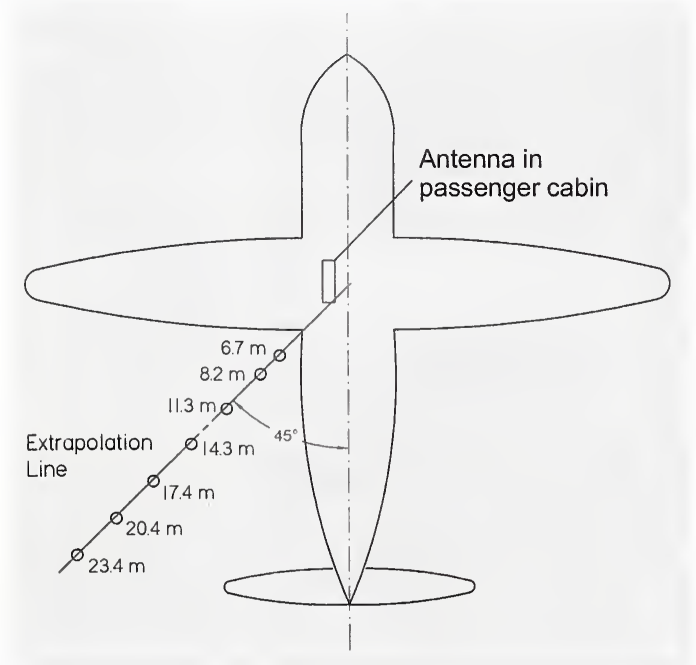

Figure 26. Extrapolation line measurement position. 


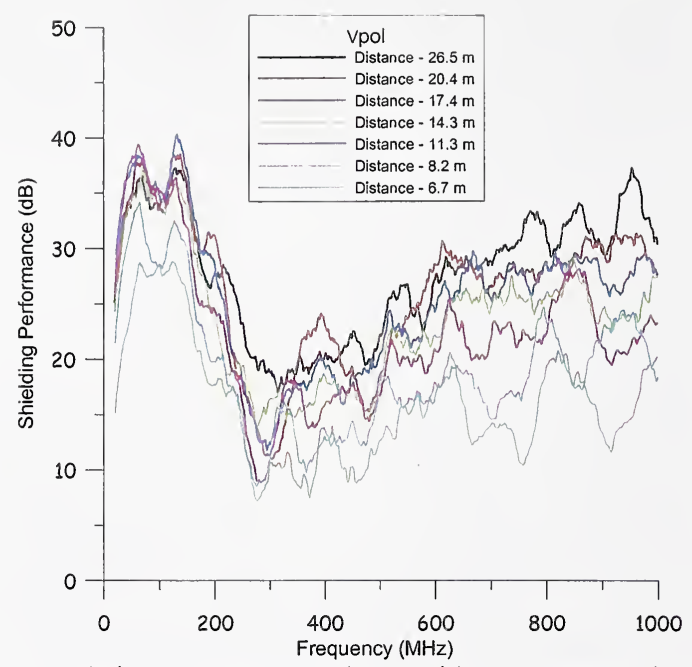

Figure 27. Plot of extrapolation measurements shown with the reference distance fixed at $\mathrm{D}=4.24 \mathrm{~m}$-vertical polarization, $50 \mathrm{MHz}$ averaging bandwidth. Note the large spread in data above $200 \mathrm{MHz}$.

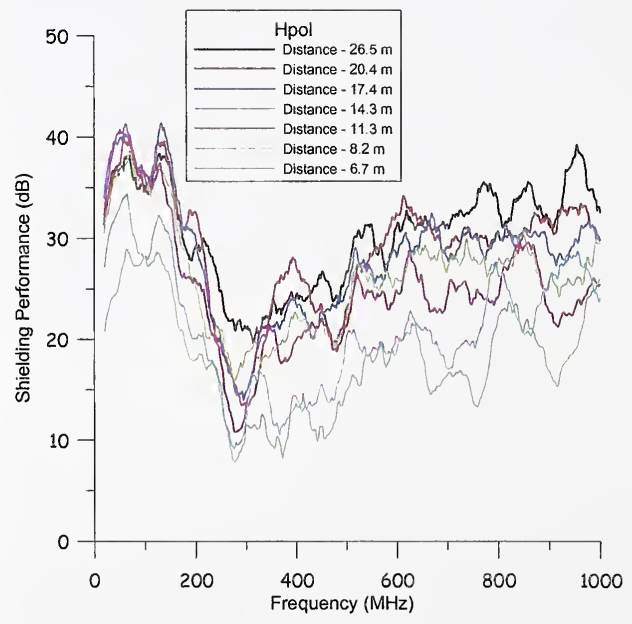

Figure 28. Plot of extrapolation measurements shown with the reference distance fixed at $\mathrm{D}=4.24 \mathrm{~m}$-horizontal polarization, $50 \mathrm{MHz}$ averaging bandwidth. Note the large spread in data above $200 \mathrm{MHz}$. 


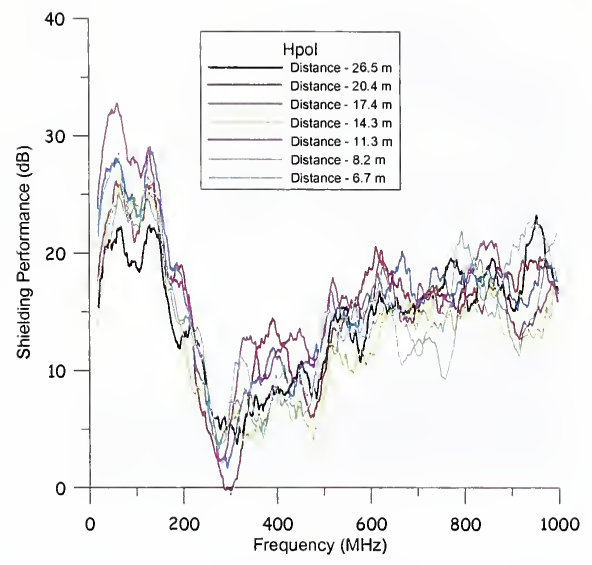

Figure 29. Shielding performance results obtained at all of extrapolation positions using the distance correction of equation 5 (horizontal polarization), $50 \mathrm{MHz}$ averaging bandwidth. Note the reduced spread in the data.

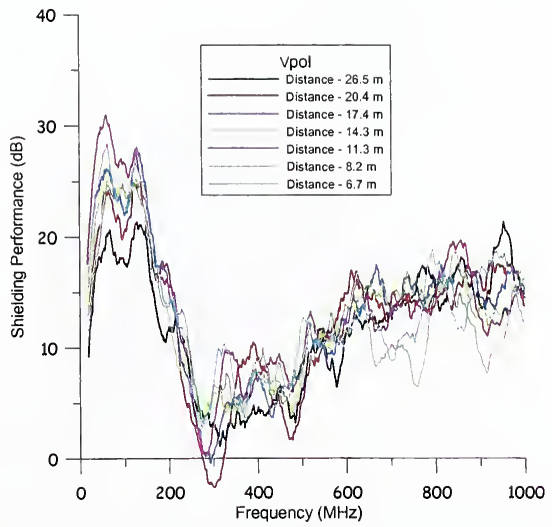

Figure 30. Shielding performance results obtained at all of extrapolation positions using the distance correction of equation 5 (vertical polarization), $50 \mathrm{MHz}$ averaging bandwidth. Note the reduced spread in the data. 


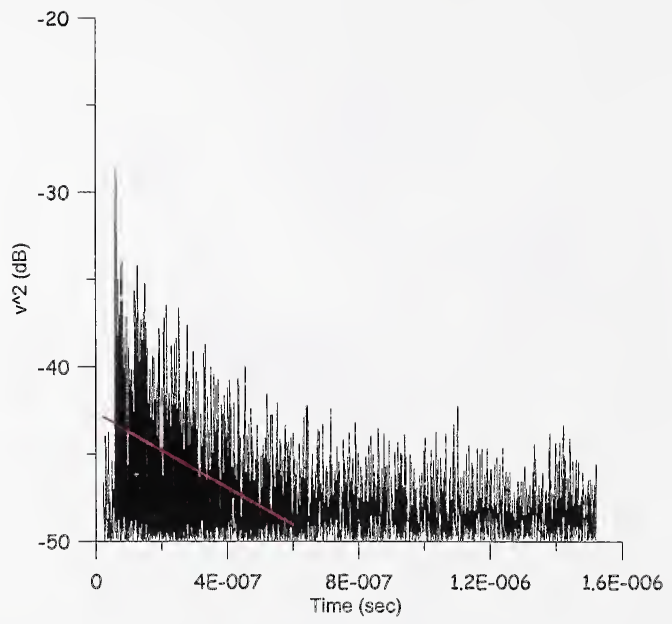

Figure 31. Amplitude-squared time-domain waveform obtained at a distance of $26.5 \mathrm{~m}$ along the extrapolation line. The straight line is obtained from a linear least-squares fit to a $600 \mathrm{~ns}$ segment of the data.

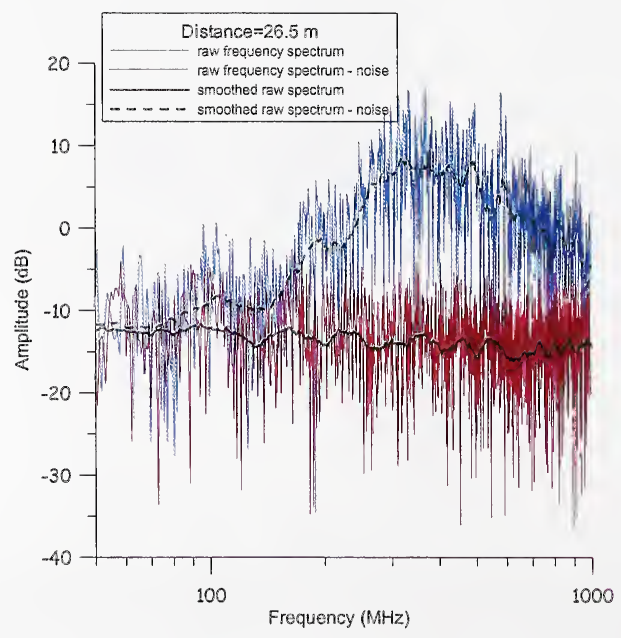

Figure 32. A comparison of the amplitude spectrum (obtained at the $26.5 \mathrm{~m}$ extrapolation position) with the system noise level. 


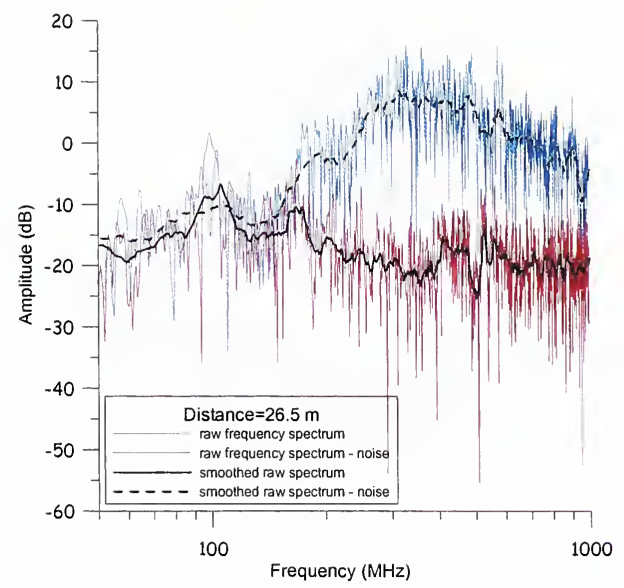

Figure 33. Gated amplitude spectrum of extrapolation position at the same $26.5 \mathrm{~m}$ extrapolation position compared with the system noise level.

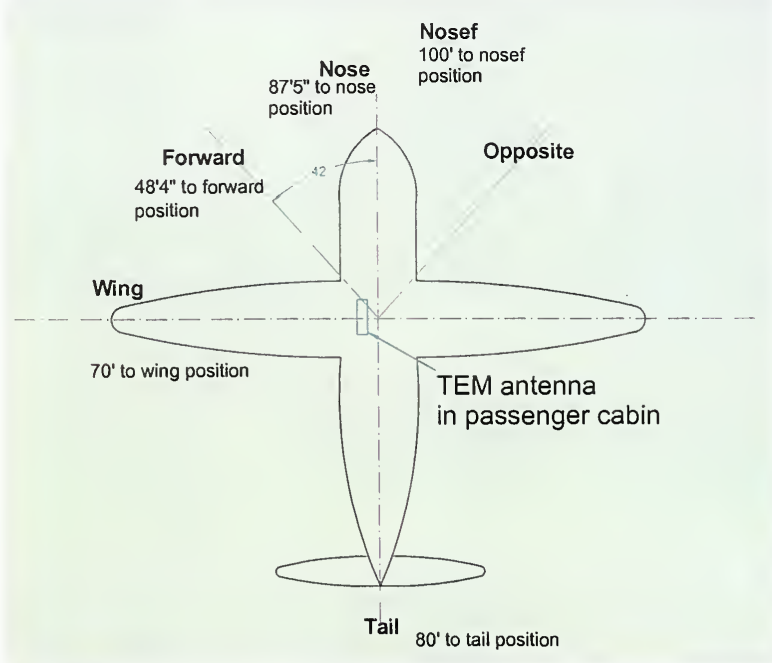

Figure 34. Measurement from passenger cabin showing all receive locations. 


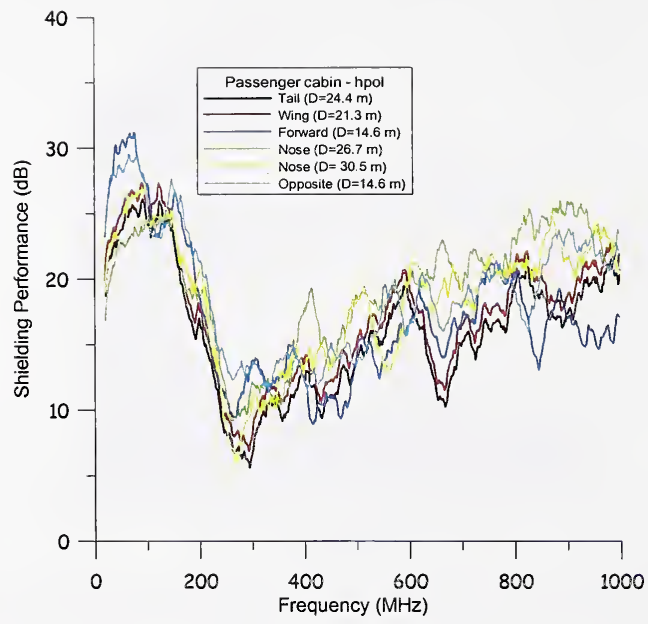

Figure 35. Shielding performance data obtained with the transmit antenna located at the aircraft center in the main passenger cabin for different receiving antenna positions and angles-horizontal polarization, $50 \mathrm{MHz}$ averaging bandwidth.

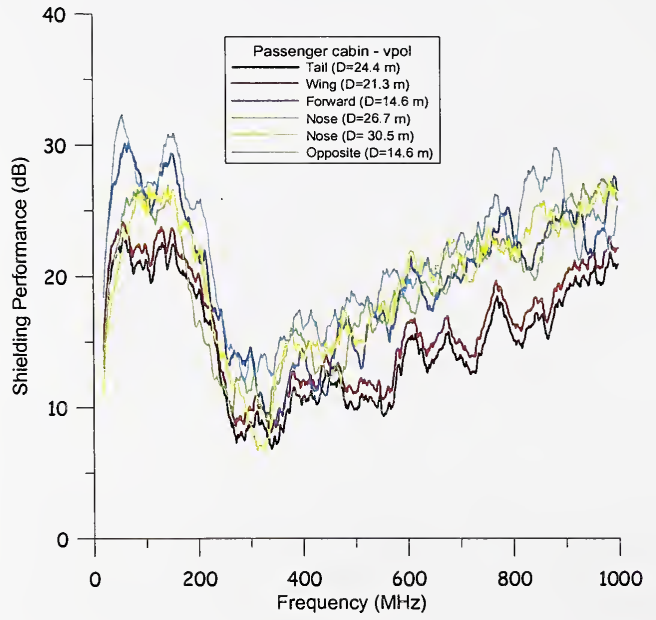

Figure 36. Shielding performance data with transmit antenna on the passenger deck-vertical polarization, $50 \mathrm{MHz}$ averaging bandwidth. 


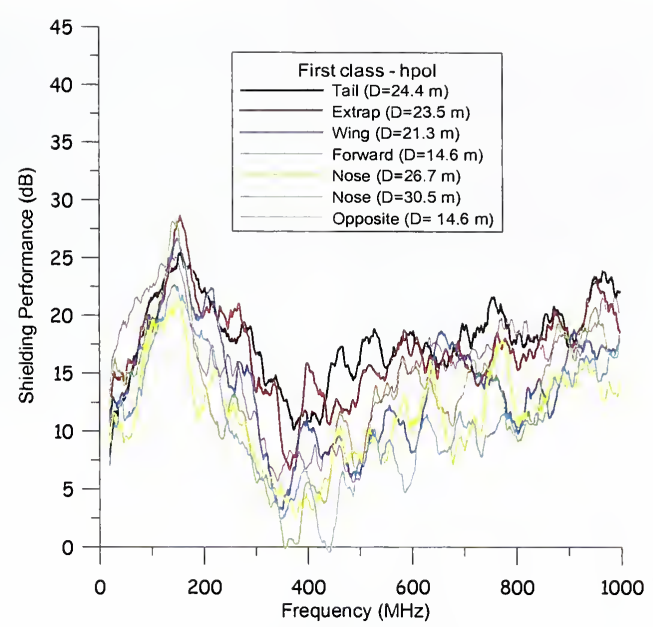

Figure 37. Shielding performance data with transmit antenna in the first class cabin-horizontal polarization, $50 \mathrm{MHz}$ averaging bandwidth.

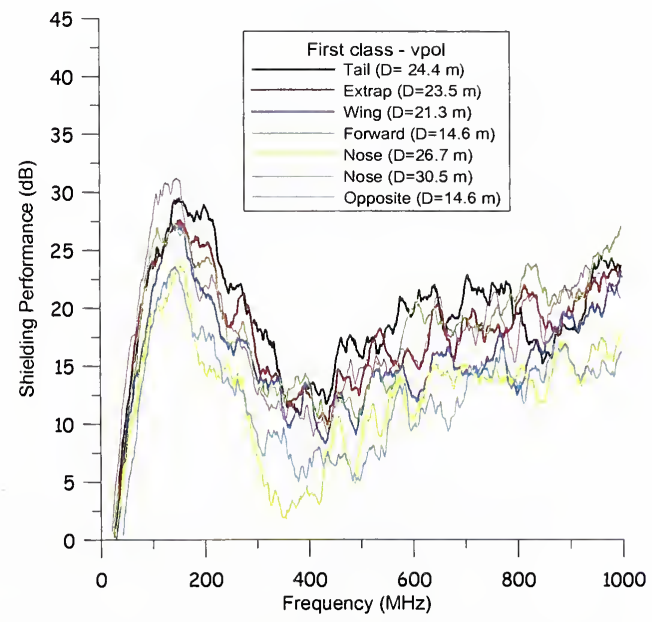

Figure 38. Shielding performance data with transmit antenna in the first class cabin-vertical polarization, $50 \mathrm{MHz}$ averaging bandwidth. 

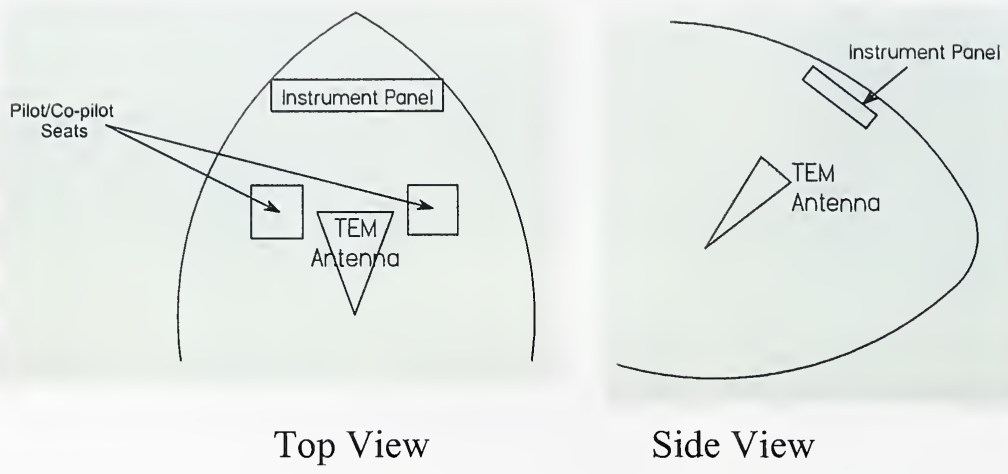

Side View

Figure 39. Transmitting TEM horn antennas configuration in the cockpit..

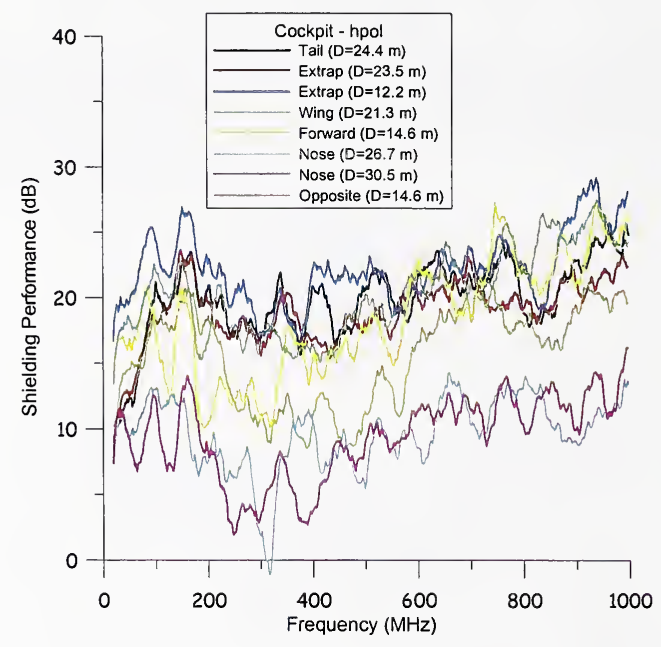

Figure 40. Cockpit shielding performance-horizontal polarization, $50 \mathrm{MHz}$ averaging bandwidth. 


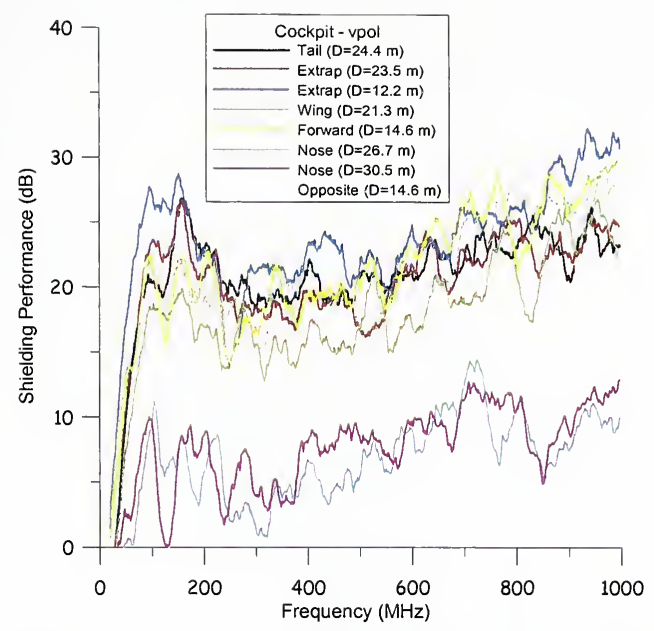

Figure 41. Cockpit shielding performance-vertical polarization, $50 \mathrm{MHz}$ averaging bandwidth.

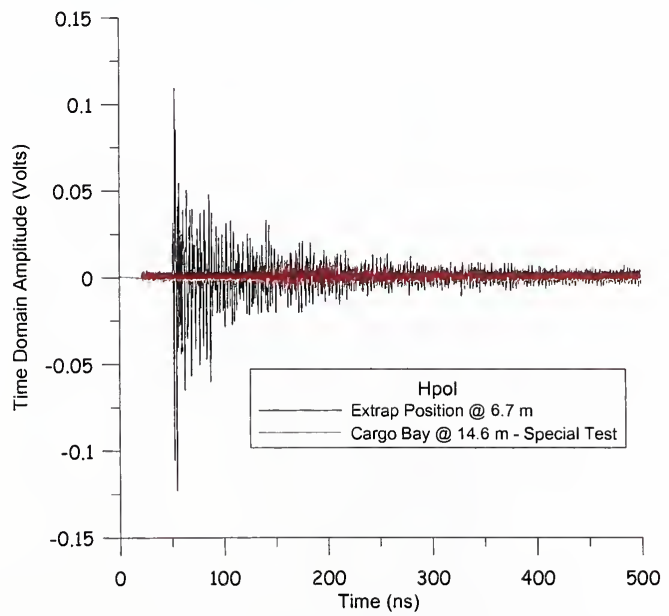

Figure 42. Received signal level comparison between extrapolation position at $6.71 \mathrm{~m}$ and the cargo bay. 


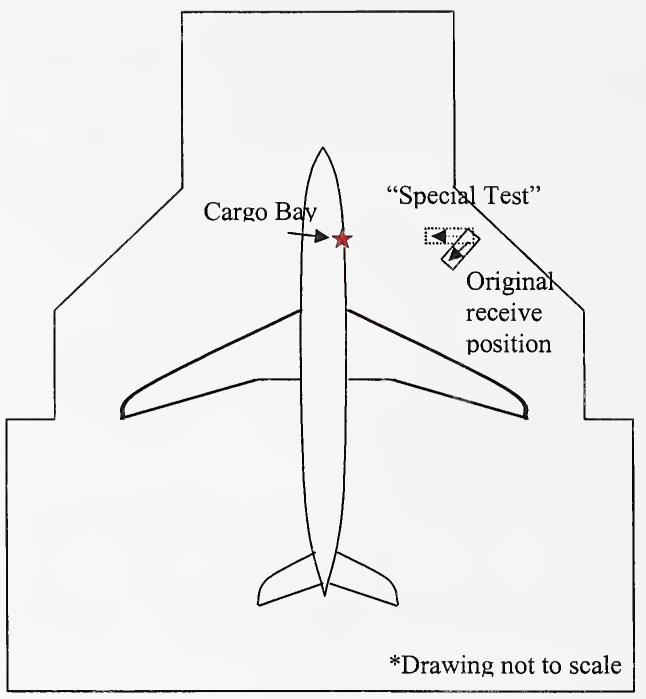

Figure 43. Measurement setup showing "special test" position.

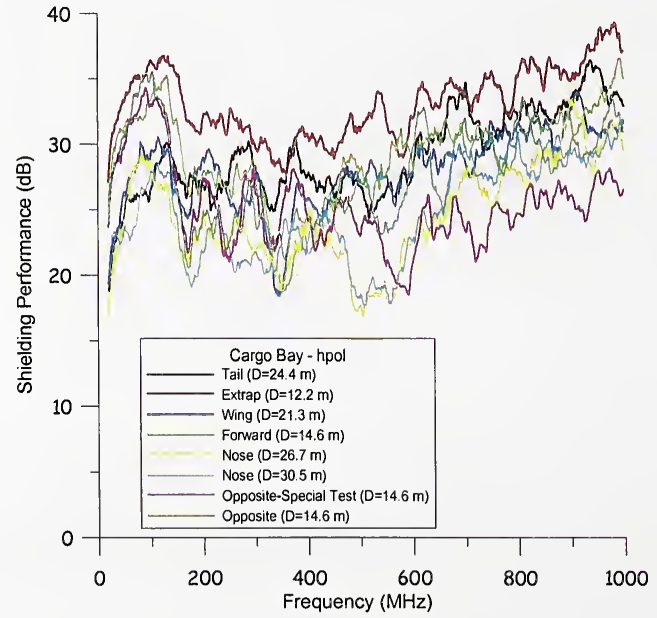

Figure 44. Cargo bay shielding performance-horizontal polarization, $50 \mathrm{MHz}$ averaging bandwidth. 


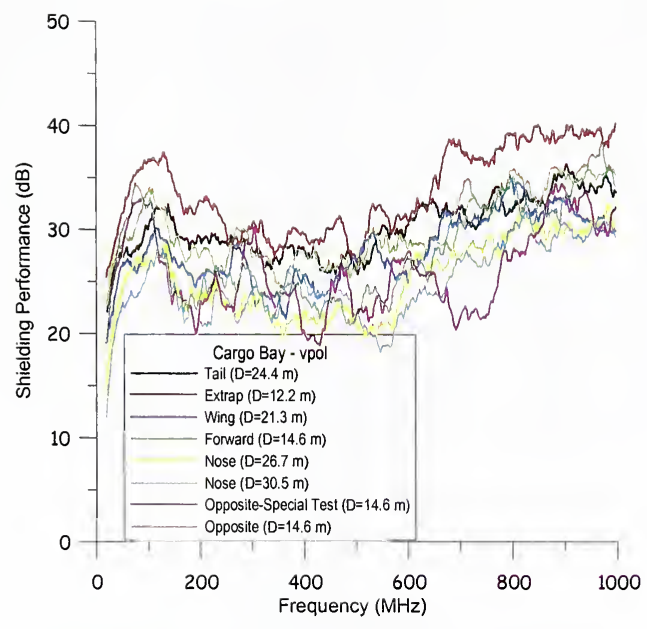

Figure 45. Cargo bay shielding performance-vertical polarization, $50 \mathrm{MHz}$ averaging bandwidth.

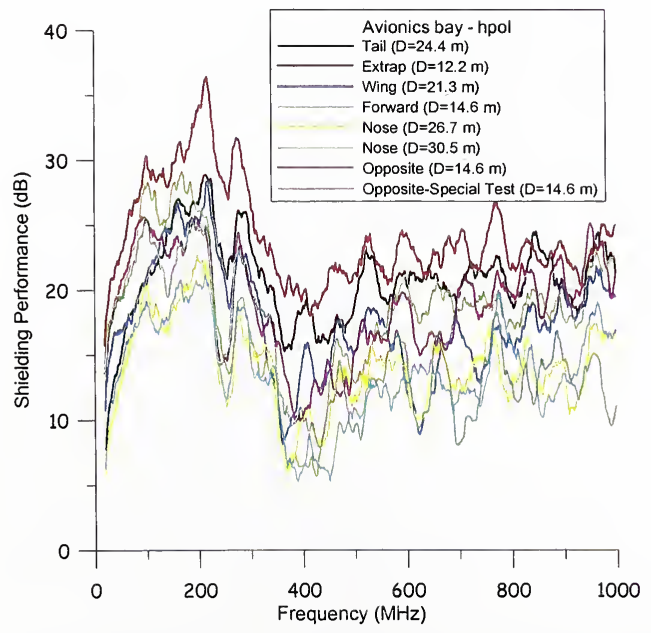

Figure 46. Avionics bay shielding performance-horizontal polarization, $50 \mathrm{MHz}$ averaging bandwidth. 


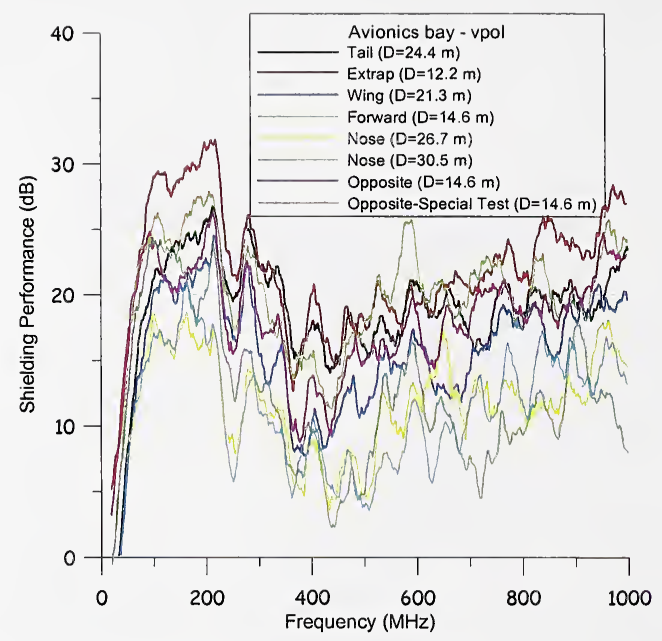

Figure 47. Avionics bay shielding performance-vertical polarization, $50 \mathrm{MHz}$ averaging bandwidth.

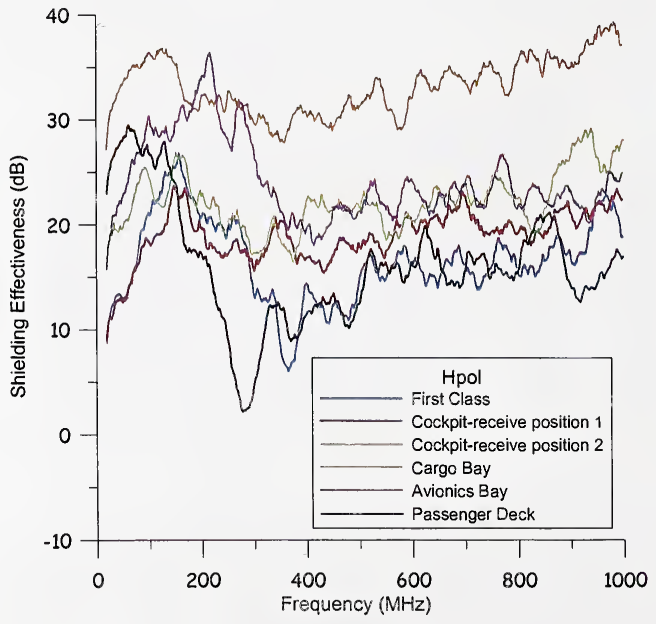

Figure 48. Shielding performance as a function of transmitter location. The receiving antennas are located at distances of $12 \mathrm{~m}$ and $23.5 \mathrm{~m}$ on the extrapolation line, $50 \mathrm{MHz}$ averaging bandwidth. 


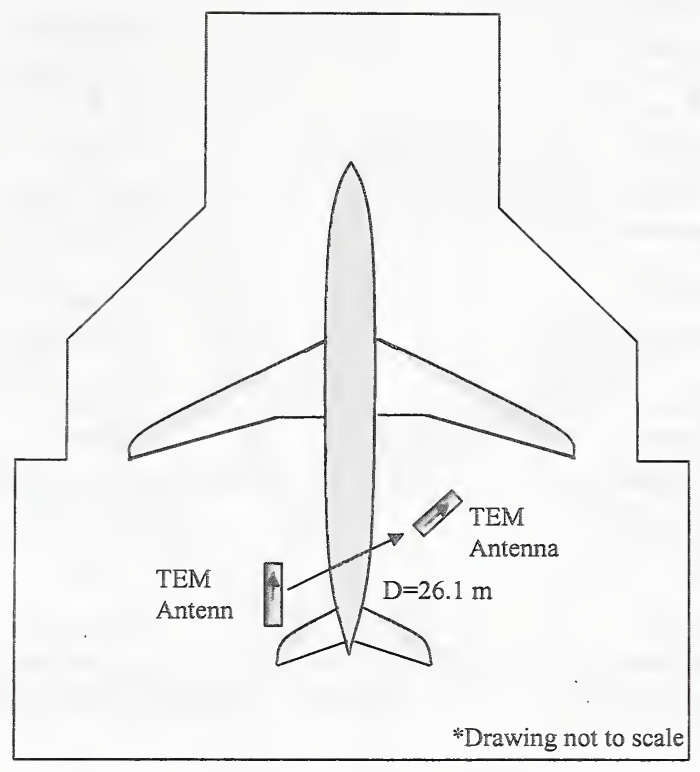

Figure 49. Measurement setup for the reverberant reference technique.

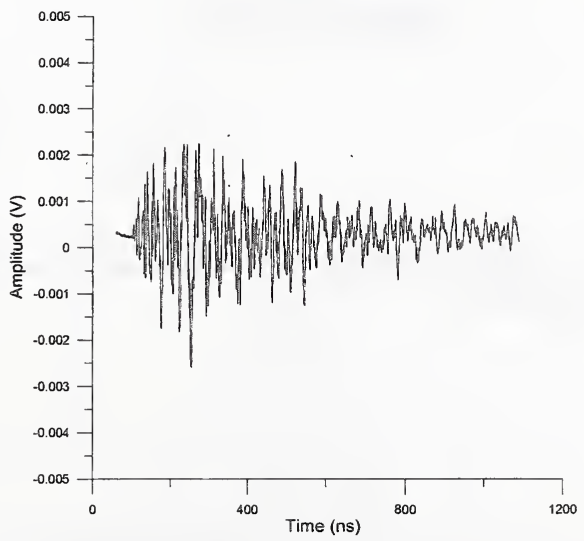

Figure 50. Reverberant reference waveform showing the hangar environmental scattering. 


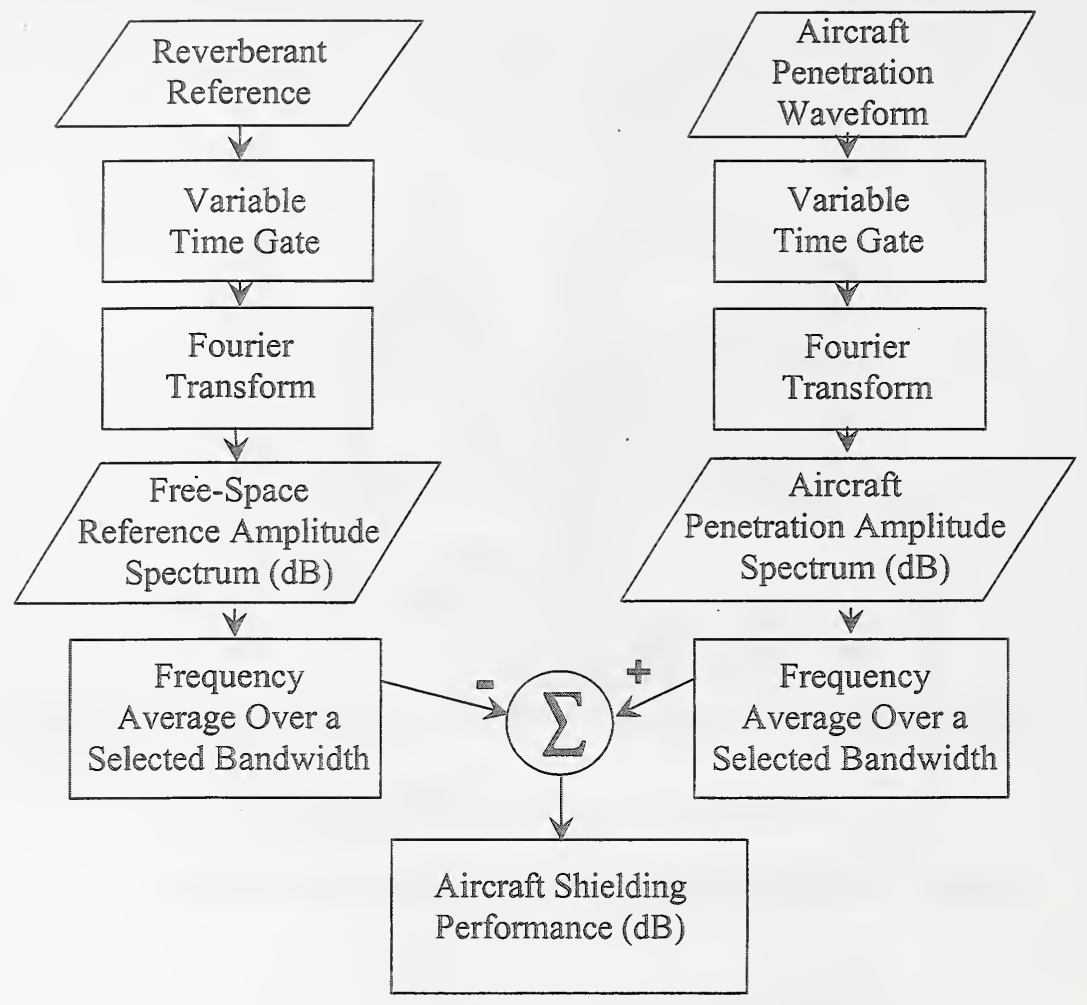

Figure 51. Signal processing sequence to obtain aircraft shielding performance for the reverberant reference technique. 


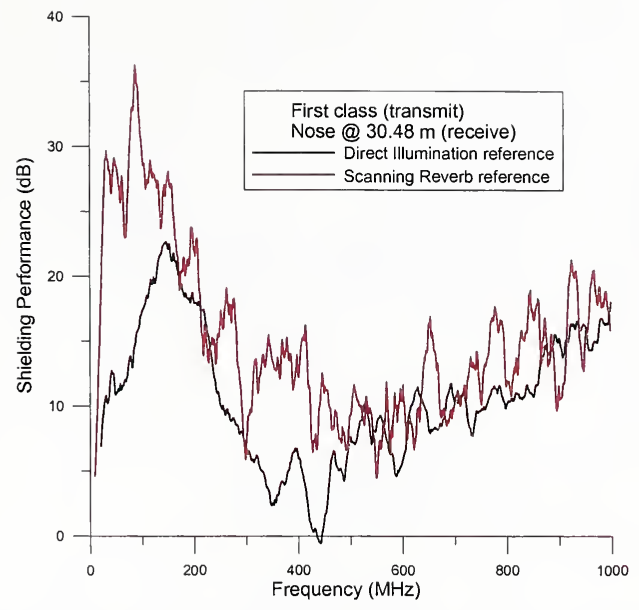

Figure 52. Comparison between scanning reverb technique and direct illumination technique horizontal polarization, $50 \mathrm{MHz}$ averaging bandwidth.

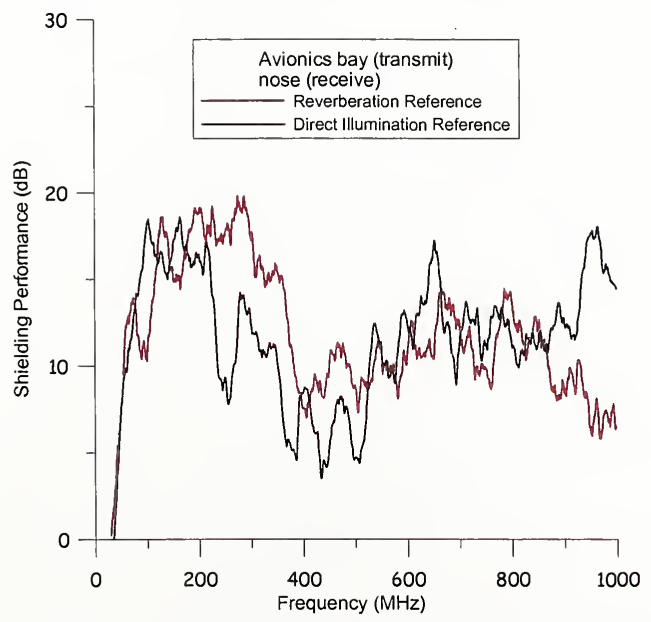

Figure 53. Comparison between reverb reference technique and direct illumination techniquehorizontal polarization, $50 \mathrm{MHz}$ averaging bandwidth. 


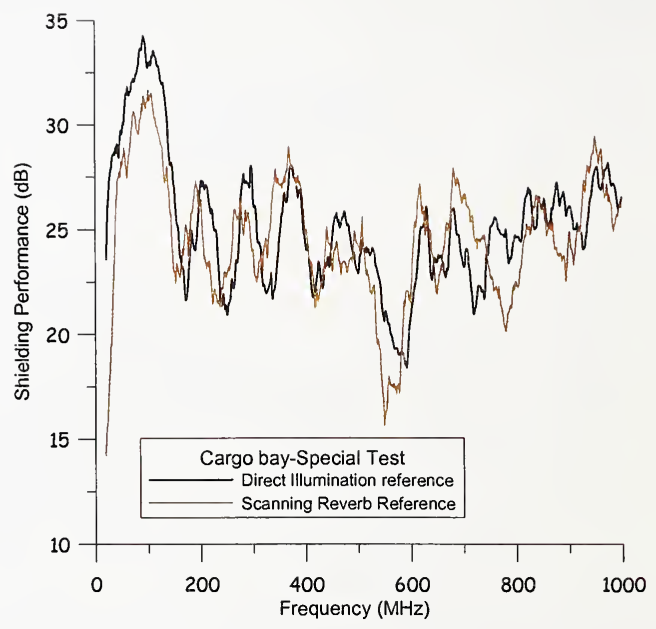

Figure 54. Comparison between references for transmit antenna in cargo bay-horizontal polarization, $50 \mathrm{MHz}$ averaging bandwidth. 
)

) 